\title{
van der Waals Interactions in Material Modelling
}

\author{
Jan Hermann and Alexandre Tkatchenko
}

\section{Contents}

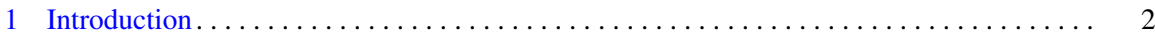

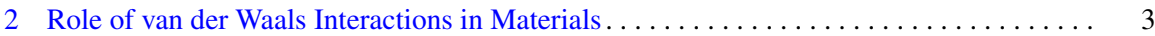

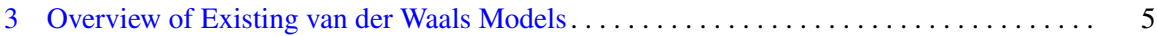

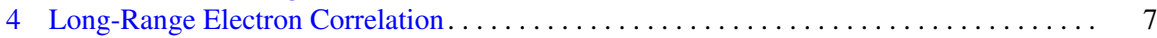

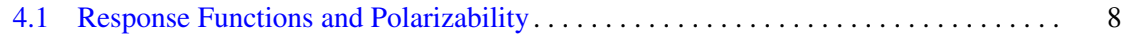

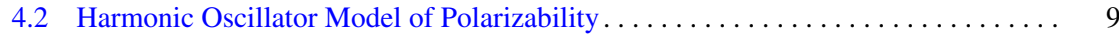

4.3 Range Separation in Density Functional Theory . . . . . . . . . . . . . . . 11

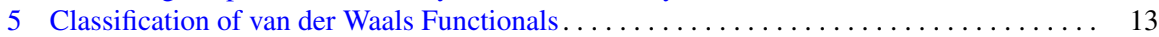

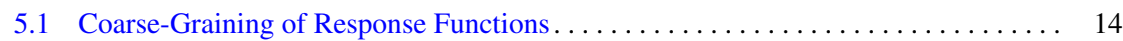

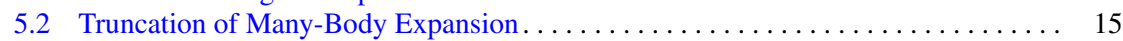

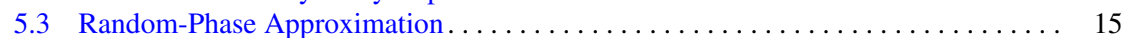

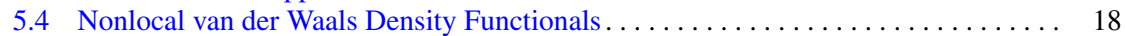

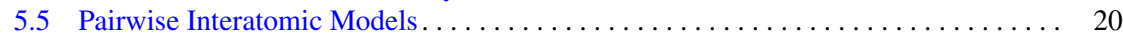

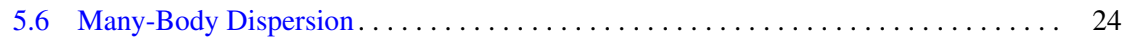

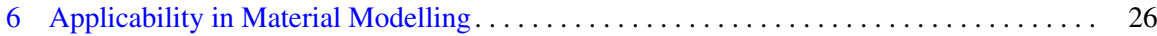

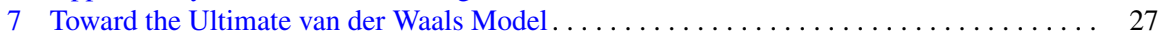

References..................................... 29

\begin{abstract}
Van der Waals (vdW) interactions stem from electronic zero-point fluctuations and are often critical for the correct description of structure, stability, and response properties of molecules and materials, including biomolecules, nanomaterials, and material interfaces. Here, we give a conceptual as well as mathematical overview of the current state of modeling vdW interactions,
\end{abstract}

\footnotetext{
J. Hermann • A. Tkatchenko ( $₫)$

Physics and Materials Science Research Unit, University of Luxembourg, Luxembourg, Luxembourg

e-mail: alexandre.tkatchenko@uni.lu
} 
focusing in particular on the consequences of different approximations for practical applications. We present a systematic classification of approximate first-principles models based on the adiabatic-connection fluctuation-dissipation theorem, namely the nonlocal density functionals, interatomic methods, and methods based on the random-phase approximation. The applicability of these methods to different types of materials and material properties is discussed in connection with availability of theoretical and experimental benchmarks. We conclude with a roadmap of the open problems that remain to be solved to construct a universal, efficient, and accurate vdW model for realistic material modeling.

\section{Introduction}

van der Waals (vdW) interactions stem from electronic zero-point (and possibly thermal) fluctuations in electronic matter (Langbein 1974; Parsegian 2005). Therefore, vdW interactions scale rapidly with system size and are often critical for the correct description of structure, stability, and response properties of molecules and materials, including biomolecules, nanomaterials, and material interfaces. This makes proper description of $\mathrm{vdW}$ interactions a crucial aspect of modern material modelling. This is especially important in the context of electronic-structure calculations using approximate density functionals. Such functionals are normally semilocal ("short sighted") in the electronic density, meaning that nonlocal vdW interactions are poorly or not at all described by these functionals.

From a classical electrostatic perspective, electrons repel each other. However, quantum-mechanical correlation effects typically act to minimize the electric repulsion between electrons. This correlation in the electronic motion results in instantaneous effective multipoles that interact via electrostatic forces, attracting different regions toward each other. When there is no significant overlap between two interacting regions of electronic matter, we usually speak of the long-range electron correlation, which is the underlying microscopic cause of vdW interactions. (As Margenau put it already in 1939, "the term 'van der Waals force' is not one of very precise usage," which holds to this date. Here, we will use the term exclusively to refer to the electron correlation part of noncovalent interactions.)

In many approaches to material modelling, ranging from empirical to those based on first principles, the models that describe the short-range and long-range parts of the electron correlation are constructed separately, because each requires a different set of considerations. This chapter discusses currently used approaches to the calculation of the long-range correlation energy - the vdW energy - with special focus on the case when the short-range part of the correlation energy (and other total energy components) is calculated within the density functional theory (DFT). We begin with a conceptual understanding and importance of vdW interactions in materials (Sect. 2), which has motivated the recent rapid development of new vdW models presented in the subsequent sections. This is followed by a brief conceptual overview of existing and widely used vdW models (Sect. 3), which can server 
either as a stand-alone short synopsis or as an introduction to the subsequent more detailed exposition. We then continue with a more theoretical presentation of current approaches to modelling vdW interactions, first introducing a general framework for discussing vdW models (Sect. 4), followed by a classification of current approaches within that framework (Sect. 5). None of the existing vdW models is the best choice for every system, and a close attention must be paid to their accuracy and areas of applicability (Sect. 6). We conclude with an outline of outstanding problems in the topic of modelling vdW interactions from first principles of quantum mechanics (Sect. 7).

\section{Role of van der Waals Interactions in Materials}

Functional materials are becoming increasingly smaller in size and more heterogeneous in composition. These two aspects of novel nanomaterials lead to the emergence of nontrivial quantum-mechanical effects that depend on size and topology and which may ultimately determine the properties of a material of interest. One important consequence of this evolution beyond traditional materials, the functionality of which was largely regulated by bulk observables, is that noncovalent interactions play an increasingly important role in determining the structure, stability, and ensuing function of homogeneous and heterogeneous nanostructured materials. van der Waals interactions, which exhibit nontrivial scaling behavior with system size (Dobson et al. 2006; Ruzsinszky et al. 2012; Gobre and Tkatchenko 2013), are often the dominant part of such noncovalent interactions. In general, $\mathrm{vdW}$ interactions have already been recognized as playing an instrumental role in determining the structure, stability, and functionality of biological materials, supramolecular and sensor chemistry, pharmaceuticals, dye-sensitized solar cells, and many other systems. More recently, the field of "van der Waals heterostructures" has moved into the forefront (Geim and Grigorieva 2013) and has already led to fundamental advances in the study of low-dimensional materials and to a number of novel technological applications.

In this context, the importance of understanding and accurately modelling vdW interactions in realistic materials can hardly be overemphasized. However, our ability to model these ubiquitous quantum mechanical effects has been severely impeded by the prohibitively high computational cost of explicitly correlated quantum chemical methods and the lack of efficient approximations to the manyelectron correlation problem for large systems (Szabo and Ostlund 1996). In fact, most successful approximations employed for modelling vdW interactions in materials rely on the rather crude lowest-order pairwise additive approximation, which is only exact in the weak polarization limit and at large interatomic distances. For condensed-phase systems with a moderate to large polarizability density, such pairwise approximations can often lead to qualitatively incorrect predictions of structural, energetic, and response properties.

The structure and binding in organic materials is often driven by vdW interactions, in combination with other contributions such as repulsion, electrostatics, 
and induction. Therefore, accurate modelling of $\mathrm{vdW}$ interactions is critical for understanding the properties of organic materials. In recent years, substantial progress has been achieved in the theoretical prediction of structures and stabilities of molecular crystals by using vdW-inclusive DFT approaches. Today, the structures of (simple) organic molecular crystals can be predicted with an accuracy of 2-3\% and cohesive energies to $1-2 \mathrm{kcal} / \mathrm{mol}$. Proper description of $\mathrm{vdW}$ correlations becomes even more relevant when one looks at the relative energetics of molecular systems, which are essential to predict the polymorphic behavior of molecular crystals.

While the crucial role of vdW interactions in organic materials is well established, our understanding of the relative importance of these ubiquitous interactions in semiconductors, ionic solids, and metals is still in development. The contribution of the long-range $\mathrm{vdW}$ energy to the cohesive energy of elemental and binary semiconductors and ionic solids amounts to $0.2-0.3 \mathrm{eV} /$ atom, which is around $8 \%$ of the cohesive energy (Zhang et al. 2011). The contribution of vdW energy to the bulk modulus is even more pronounced, reaching up to $22 \%$ for Ge and GaAs. Notably, the inclusion of vdW interactions in DFA calculations allows to simultaneously improve the performance for lattice constants, cohesive energies, and bulk moduli, when compared to experiment. Similar conclusions have been reached for a wide variety of hard solids. Because vdW interactions typically have larger contributions to relative energetics than absolute ones, one expects significant effects for phase transition pressures and phase diagrams of most solids.

The properties of many materials are substantially affected by the presence of simple and complex defects. For example, the properties of semiconductors are largely determined by neutral and charged interstitials and vacancies (Freysoldt et al. 2014). The formation of defects entails a modification of polarization around defect sites, and this can have a substantial effect on the contribution of vdW energy to the stability and mobility of defects. For instance, the inclusion of interactions in DFT improves the description of defect formation energies, significantly changes the barrier geometries for defect diffusion, and brings migration barrier heights into close agreement with experimental values (Gao and Tkatchenko 2013). In the case of $\mathrm{Si}$, the vdW energy substantially decreases the migration barriers of interstitials and impurities by up to $0.4 \mathrm{eV}$, qualitatively changing the diffusion mechanism. Moving beyond point defects, it is to be expected that more complex neutral and charged multiatom defects and dislocations will lead to even stronger nonlocal polarization effects and intricate dependence of $\mathrm{vdW}$ interactions on the nature of defects.

Hybrid inorganic/organic systems (HIOS) are relevant for many applications in catalysis, light-emitting diodes, single-molecule junctions, molecular sensors and switches, and photovoltaics. The predictive modelling and understanding of the structure and stability of such hybrid systems are an essential prerequisite for tuning their electronic properties and functions. The bonding in HIOS is often determined by a delicate balance between covalent bonds, hydrogen bonds, charge transfer, Pauli repulsion, and vdW interactions. Yet the latter are at the edge of what is possible with current vdW approaches, because the inorganic substrate is often a 
metal or a doped semiconductor, which is difficult to model due to the delocalized nature of metallic single-particle excitations.

\section{Overview of Existing van der Waals Models}

A common starting point for virtually all existing vdW models is an exact expression for the electron correlation energy that can be constructed from the ACFD theorem (Gunnarsson and Lundqvist 1976; Langreth and Perdew 1977). The essential idea of this expression is an interpolation between a reference noninteracting meanfield system and the fully interacting many-body system. The starting quantity from which the correlation energy can be calculated is some form of a response function (nonlocal polarizability, dielectric function, density response function), which describes the linear response of the system to external electric field. This quantity can be calculated either from first principles, using some mean-field electronic-structure theory such as the Hartree-Fock (HF) method or Kohn-Sham DFT (KS-DFT), or constructed semiempirically using approximate models. The former approach is less ambiguous, but the complexity of such ab initio response functions demands large computational cost and even then requires substantial approximations to the interelectronic interactions. In contrast, the latter approach leads to efficient methods, in which much of the complexity of the electronic structure can be treated effectively by suitable parameterization of the model response functions. In both cases, either the response functions or the interelectronic interactions (or both) are treated approximately, leading to deviations from the inprinciple exact ACFD correlation energy.

Essentially all existing methods for modelling vdW interactions can be derived by following various approximations to the ACFD theorem. Two major classes of approximations that can be identified in existing vdW models are the coarsegraining of the response functions and truncation of the many-body expansion. In their full form, the response functions are two-point spatial functions, specifying a response of a system at some point to the perturbation in the electric field in another point. Such a description enables the calculation of the electron correlations across the whole range of interelectronic distances, including the short-range intra-atomic as well as long-range intermolecular correlations. This level of detail is largely unnecessary for the calculation of the vdW energy, which comprises only the longrange part of the electron correlation energy. Therefore, a common approach is to coarse-grain the response functions into finite-size fragments - typically atoms and to evaluate only the long-range correlations between the fragments, while the short-range intrafragment interactions are captured in the effective response properties of the fragments. The second common approximation involves the truncation of the many-body expansion. The closed-form ACFD expression can be expanded as an infinite sum of terms that involve repeated couplings of the noninteracting response functions. Ordering these terms by the number of times a response function occurs in them, the electron correlation energy starts at second order (two occurrences) and continues to infinity. The physical interpretation of the 


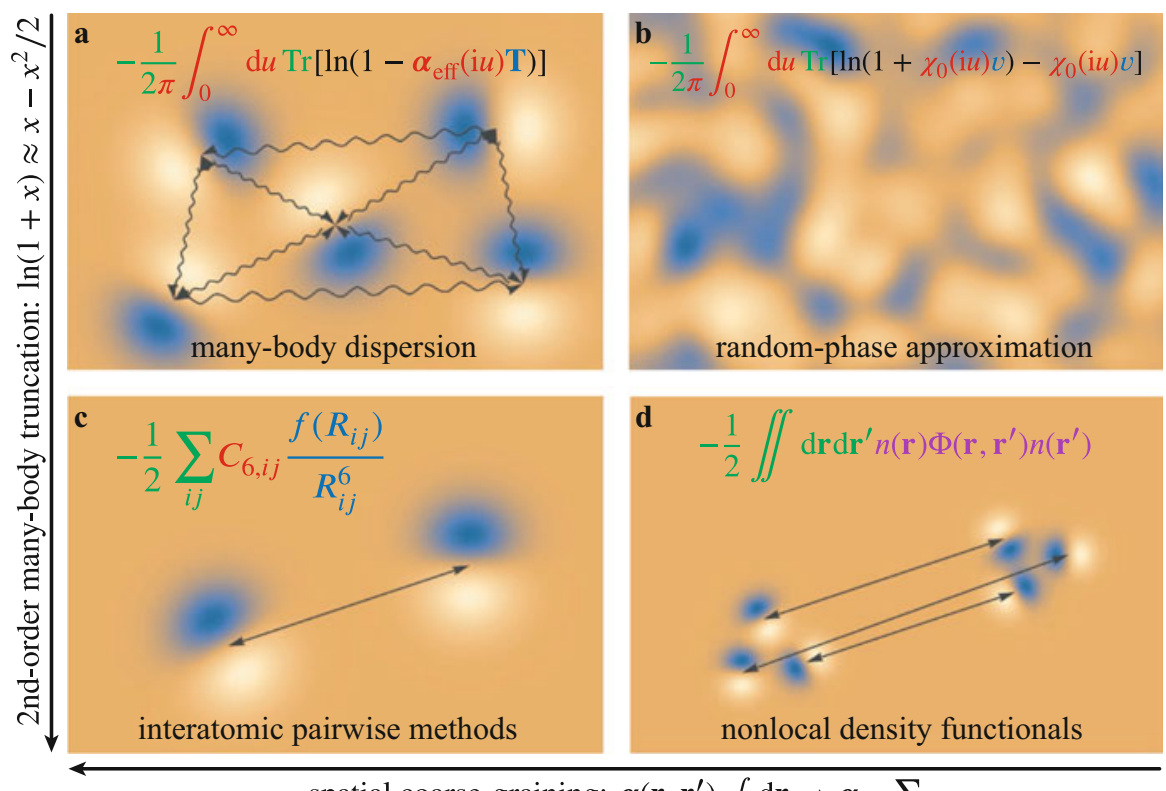

spatial coarse-graining: $\boldsymbol{\alpha}\left(\mathbf{r}, \mathbf{r}^{\prime}\right), \int \mathrm{d} \mathbf{r} \rightarrow \boldsymbol{\alpha}_{i j}, \sum_{i}$

Fig. 1 Classification of different approaches to modelling vdW interactions based on their approximations to the adiabatic-connection fluctuation-dissipation (ACFD) formula. The light yellow and blue illustrate density fluctuations, and the arrows denote interactions between them. General formulas for each method class are shown, with colors denoting parts with the same origin in the ACFD formula: summation of all interactions in the system (green), encoding of the response properties (red), and the interaction potentials (blue). In random-phase approximation (RPA), both the noninteracting frequency-dependent density response function, $\chi_{0}\left(\mathbf{r}, \mathbf{r}^{\prime}, u\right)$, and the electronic Coulomb interaction, $v\left(\mathbf{r}, \mathbf{r}^{\prime}\right)=\left|\mathbf{r}-\mathbf{r}^{\prime}\right|^{-1}$, are nonlocal spatial quantities, and their multiplication should be interpreted as $\left[\chi_{0} v\right]\left(\mathbf{r}, \mathbf{r}^{\prime}, u\right)=\int \mathrm{d} \mathbf{r}^{\prime \prime} \chi_{0}\left(\mathbf{r}, \mathbf{r}^{\prime \prime}, u\right) v\left(\mathbf{r}^{\prime \prime}, \mathbf{r}^{\prime}\right)$. The RPA itself neglects the exchange-correlation screening of the Coulomb interaction and is not illustrated in this figure. In many-body dispersion (MBD), the response function and potential are coarse-grained and expressed as the nonlocal polarizability matrix, $\boldsymbol{\alpha}_{i j}(u)$, and dipole matrix, $\mathbf{T}_{\mathrm{ij}}$, which are multiplied as ordinary matrices. Nonlocal functionals truncate the many-body expansion (expressed as the logarithm in RPA and MBD) at second order, and the response function and interaction potential are intermingled (violet) in the nonlocal kernel, $\Phi\left(\mathbf{r}, \mathbf{r}^{\prime}\right)$. Interatomic pairwise models, coarsegrained and truncated at second order, integrate the frequency-dependent polarizability into the so-called $C_{6}$ coefficients, while the square of the dipole potential yields the $R^{-6}$ power law

$n$-th order is such that $n$ electronic fluctuations interact via the long-range Coulomb potential to yield a contribution to the correlation energy. The magnitude of the contribution of the individual orders to the vdW energy decreases with the growing order, which motivates the common approximation of truncating the infinite sum at some order, usually second or third. Using only the lowest second order results in the large class of pairwise methods, which neglect any many-body effects in vdW interactions. 
Organizing existing vdW models into a 2-by-2 matrix based on whether they use the coarse-graining and/or the many-body truncation approximations (Fig. 1) provides a concise classification that allows for an efficient discussion of the general behavior of these models. The class of methods that do not coarse-grain nor truncate the many-body expansion are based exclusively on the ab initio noninteracting response functions obtained from the HF or KS-DFT methods. This requires other approximations to the interelectronic interactions in the ACFD formula, which make its evaluation tractable (though still computationally demanding). The most straightforward of these approaches is the so-called random-phase approximation (RPA), in which the complex interaction between electronic fluctuations is replaced with the bare Coulomb potential. In contrast, the other three vdW model classes use model noninteracting response functions (usually polarizability), in which the electronic fluctuations are usually assumed to be localized. In nonlocal density functionals, the response function is modelled as a semilocal polarizability functional of the electron density, which is coupled between all pairs of points in space, resulting in a double spatial integral. The many-body dispersion (MBD) approach models the response of atomic fragments as that of harmonic oscillators, which enables efficient evaluation of the ACFD formula without any truncation of the many-body expansion. Finally, interatomic pairwise models use both the coarse-graining and the truncation to provide a particularly simple formula for the vdW energy, in which the response functions are expressed in the form of the so-called dispersion $C_{6}\left(C_{8}\right.$, $C_{10}, \ldots$ ) coefficients.

Within each of the four classes of vdW models, there are multiple instances that differ in more subtle details. These include the particular parametrization of the model response functions or the mechanisms that separate the short-range and longrange parts of the electron correlation. These differences influence the performance of the individual methods but do not change their general behavior for different types of molecules and materials, which will be discussed in more detail in Sect. 6. These individual models together with a more rigorous mathematical formulation of this overview are presented in the following two sections.

\section{$4 \quad$ Long-Range Electron Correlation}

The ACFD theorem yields an in-principle exact expression for the electron correlation energy and serves as a basis for the various approximate vdW models discussed in this chapter. The present and the following sections give a brief introduction to the mathematical formulation of this topic, which can be found in greater detail elsewhere (Hermann et al. 2017). The ACFD formula expresses the vdW energy in terms of the response of an electronic system to an external electric field, which is introduced in the remainder of this section, while the formula itself and the various approximations to it are discussed in the following section. 


\subsection{Response Functions and Polarizability}

The polarization of electronic matter under the influence of a time-periodic external electric field, $\mathbf{E}_{\Delta}=-\nabla v_{\Delta}$, with a given frequency, $u$, can be expressed by the change in the electron density, $\Delta n$, from the unpolarized state $\left(\mathbf{E}_{\Delta}=0\right)$. In the linear regime, this change is related to the corresponding potential, $v_{\Delta}$, via the density response function,

$$
\Delta n(\mathbf{r}, u)=\int \mathrm{d} \mathbf{r}^{\prime} \chi\left(\mathbf{r}, \mathbf{r}^{\prime}, u\right) v_{\Delta}\left(\mathbf{r}^{\prime}, u\right)
$$

Alternatively, the polarization state can be described by the polarization density, $\mathbf{P}$, which can be interpreted as a dipole density and which gives the polarized charge density via divergence,

$$
\Delta n(\mathbf{r}, u)=\nabla \cdot \mathbf{P}(\mathbf{r}, u)
$$

The polarization density is related to the electric field via the (nonlocal) dipole polarizability, $\boldsymbol{\alpha}$, (Hunt 1983),

$$
\mathbf{P}(\mathbf{r}, u)=-\int \mathrm{d} \mathbf{r}^{\prime} \boldsymbol{\alpha}\left(\mathbf{r}, \mathbf{r}^{\prime}, u\right) \mathbf{E}_{\Delta}\left(\mathbf{r}^{\prime}, u\right)
$$

In general, the response of the electron density is anisotropic, $\mathbf{E}_{\Delta}$ and $\mathbf{P}$ are not aligned, and the polarizability must be a tensor. The relation between the density response function and dipole polarizability is obtained by taking the divergence of Eq. 3, integrating by parts, using the definitions of $\mathbf{E}_{\Delta}$ and $\mathbf{P}$, and comparing to Eq. 1 ,

$$
\begin{gathered}
\chi\left(\mathbf{r}, \mathbf{r}^{\prime}, u\right)=-\nabla \cdot \nabla^{\prime} \cdot \boldsymbol{\alpha}\left(\mathbf{r}, \mathbf{r}^{\prime}, u\right) \\
=-\sum_{\iota \zeta} \frac{\partial^{2}}{\partial r_{\iota} \partial r_{\zeta}^{\prime}} \alpha_{\iota \zeta}\left(\mathbf{r}, \mathbf{r}^{\prime}, u\right) \quad(\iota, \zeta=x, y, z)
\end{gathered}
$$

Whereas the electron density and the density response functions interact electrically via the Coulomb operator, the polarization density and dipole polarizability interact via the dipole operator,

$$
\mathbf{T}(\mathbf{R})=\left.\nabla \otimes \nabla^{\prime} v\left(\left|\mathbf{r}-\mathbf{r}^{\prime}\right|\right)\right|_{\substack{\mathbf{r}=\mathbf{R} \\ \mathbf{r}^{\prime}=\mathbf{0}}}=\frac{-3 \mathbf{R} \otimes \mathbf{R}+R^{2} \mathbf{I}}{R^{5}}
$$

For instance, the electrostatic Coulomb self-interaction of $\Delta n$, with its corresponding $\mathbf{P}$, can be expressed in two equivalent ways,

$$
\begin{gathered}
J[\Delta n]=\frac{1}{2} \iint \mathrm{d} \mathbf{r}_{1} \mathrm{~d} \mathbf{r} \Delta n\left(\mathbf{r}_{1}\right) v\left(\left|\mathbf{r}_{1}-\mathbf{r}_{2}\right|\right) \Delta n\left(\mathbf{r}_{2}\right) \\
=\frac{1}{2} \iint \mathrm{d} \mathbf{r}_{1} \mathrm{~d} \mathbf{r}_{2} \mathbf{P}\left(\mathbf{r}_{1}\right) \cdot \mathbf{T}\left(\mathbf{r}_{1}-\mathbf{r}_{2}\right) \mathbf{P}\left(\mathbf{r}_{2}\right)
\end{gathered}
$$


The description of the response via the density response function and dipole polarizability is equivalent, and likewise the ACFD formula can be expressed using both. The density response functions are directly accessible from ab initio electronic-structure calculations, whereas the dipole polarizability is better suited for the formulation of approximate models. The reason for this is that the density response function has a complex nodal structure, as it describes depletion of the electron density at some points and its accumulation elsewhere. In contrast, the corresponding polarizability is a smooth rotation-free vector field which encodes that underlying nodal structure implicitly in terms of its local behavior via the divergence operators in Eq. 4. This is true even in the case of a delocalized density response that is characteristic of gapless or near-gapless systems. Therefore, the strength of the response is translated directly into the magnitude of the polarizability, whereas it is translated only indirectly into the magnitude of the gradient of the density response function.

For illustration, consider two one-dimensional (1D) Gaussian charge densities located at \pm 1 (as crude model of atoms) and two model density response functions, local and nonlocal (Fig. 2). In one dimension, the dipole polarizability is a scalar and uniquely determined by integrating over the density response function,

$$
\alpha^{1 \mathrm{D}}(x, y)=\int_{-\infty}^{x} \mathrm{~d} x^{\prime} \int_{-\infty}^{y} \mathrm{~d} y^{\prime} \chi^{1 \mathrm{D}}\left(x^{\prime}, y^{\prime}\right)
$$

Even in these trivial models, the density response function changes sign around atoms and has a nontrivial nodal structure, whereas the polarizability is positive everywhere. Furthermore, the delocalized density response translates into a polarizability that is still localized but over a larger region spanning both atoms.

\subsection{Harmonic Oscillator Model of Polarizability}

When the dipole polarizability is localized, as in the examples in the previous section, it can be relatively accurately represented as an effective local polarizability, $\alpha^{\text {eff }}(\mathbf{r}, u)$, formally obtained by integrating over some neighborhood, $M(\mathbf{r})$, around each point, $\mathbf{r}$,

$$
\boldsymbol{\alpha}^{\mathrm{eff}}\left(\mathbf{r}, \mathbf{r}^{\prime}, u\right) \approx \delta\left(\left|\mathbf{r}-\mathbf{r}^{\prime}\right|\right) \int_{M(\mathbf{r})} \mathrm{d} \mathbf{r}^{\prime \prime} \boldsymbol{\alpha}\left(\mathbf{r}, \mathbf{r}^{\prime \prime}, u\right) \equiv \delta\left(\left|\mathbf{r}-\mathbf{r}^{\prime}\right|\right) \boldsymbol{\alpha}^{\mathrm{eff}}(\mathbf{r}, u)
$$

A wide variety of approaches to model the spatial dependence of $\boldsymbol{\alpha}^{\text {eff }}$ exist, which will be discussed in the following sections.

The frequency dependence, on the other hand, is very often modelled by that of a charged harmonic oscillator (HO) - a Drude oscillator. Having been Fourier-transformed from the time domain, the frequency-dependent polarizability is a complex-valued function, with the real and imaginary parts encoding the 


\section{Delocalized response}
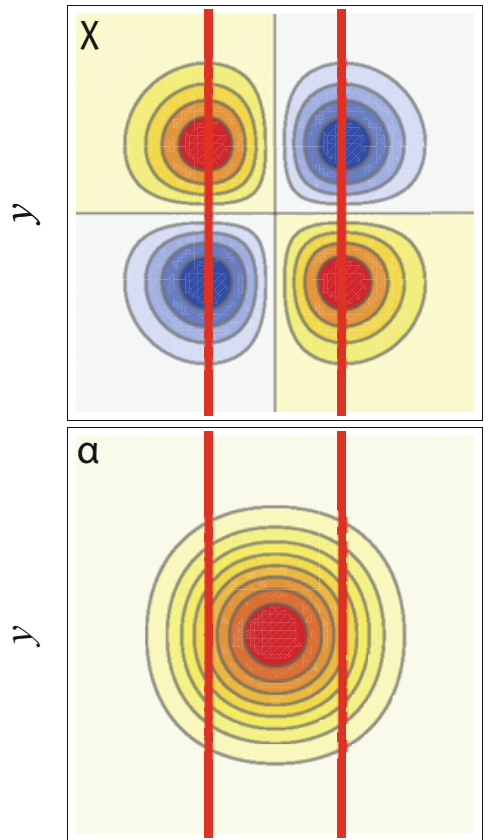

$x$
Localized response
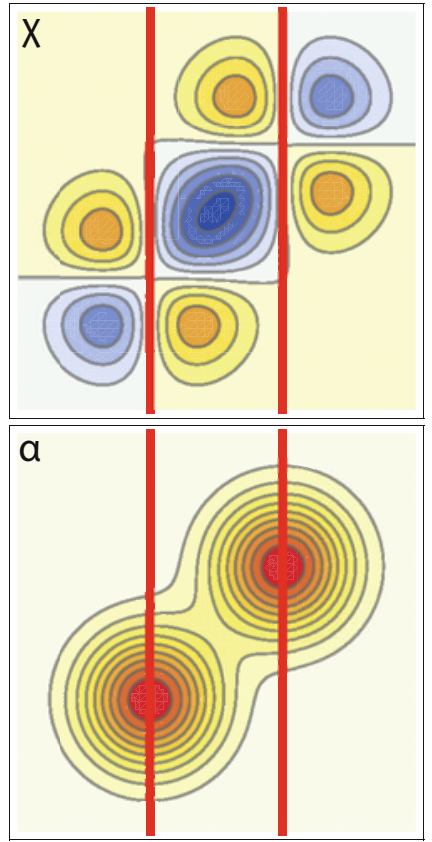

$x$

Fig. 2 Density response function, $\chi(x, y)$, compared to nonlocal dipole polarizability, $\alpha(x, y)$. The figure considers two different model one-dimensional two-particle systems with particle coordinates $x$ and $y$. The left and right systems have a delocalized and localized response, respectively. The columns encode the response of the two systems in two different but equivalent ways - as a density response function (top), $\chi(x, y)$, and as a nonlocal dipole polarizability (bottom), $\alpha(x, y)$. The red and blue colors correspond to positive and negative values. The red lines denote the positions of the two responding Gaussian charge densities on the $x$-axis

nondissipative and dissipative parts of the response, respectively. The frequency dependence of the imaginary part of the polarizability encodes the full optical (electromagnetic) absorption spectrum. This is equivalent to knowing the full energy spectrum of the corresponding Hamiltonian, which is a much harder problem than calculating the ground-state energy. Accordingly, the ACFD formula for the electron correlation energy contains the polarizability only under the integral sign over all frequencies, and it is sufficient to model the spectrum only such that its sum total is represented accurately, which is conveniently achieved with the so-called Wick rotation,

$$
\int_{0}^{\infty} \mathrm{d} u \operatorname{Im} \alpha(u)=\int_{0}^{\infty} \mathrm{d} u \alpha(\mathrm{i} u) .
$$


In contrast to the full absorption spectrum, $\operatorname{Im} \alpha(u)$, which is a complicated and highly system-dependent function of the frequency, the imaginary-axis part of $\alpha, \alpha(\mathrm{i} u)$, is a real-valued monotonically decreasing function, which has the same general shape for all kinds of systems. Together with the localized nature of the polarizability, this justifies the use of the harmonic oscillator to model the frequency dependence of the polarizability of more complex electronic systems. The polarizability of a harmonic oscillator is a simple function with two parameters, the static polarizability, $\boldsymbol{\alpha}_{0}$, and the resonance frequency, $\omega$,

$$
\boldsymbol{\alpha}^{\mathrm{HO}}(\mathrm{i} u)=\frac{q^{2} \omega^{2}}{m\left(\omega^{2}+u^{2}\right)} \equiv \frac{\boldsymbol{\alpha}_{0} \omega^{2}}{\omega^{2}+u^{2}}
$$

Consider a system of nonoverlapping bodies of electronic matter interacting via the usual electronic Coulomb force (described equivalently either by the Coulomb or dipole potential). Putting aside the internal structure of these bodies for now, their interaction energy, $E_{\text {int }}$, consists of the electrostatic part (including induction) and the (long-range) electron correlation part, $E_{\mathrm{c}}-$ the vdW energy. The ACFD formula for $E_{\mathrm{c}}$ derives its name from two parts: first, the correlations in the electronic fluctuations can be expressed in terms of the response of the bodies to an external field via the so-called fluctuation-dissipation theorem (Landau and Lifschitz 1980, sec. $§ 124)$. Second, $E_{\text {int }}$ is the difference in the total energy of the system when the Coulomb potential between the interacting bodies is switched on $(\lambda=1)$ and switched off $(\lambda=0)$. This difference can be alternatively expressed as an integral over from 0 to 1 while keeping the system at its ground state for all $\lambda$, in this way adiabatically connecting the noninteracting and interacting cases. Putting these two parts together, the ACFD theorem gives $E_{\mathrm{c}}$ in terms of the total polarizability of the nonoverlapping subsystems,

$$
-\frac{1}{2 \pi} \int_{0}^{\infty} \mathrm{d} u \iint \mathrm{d} \mathbf{r} \mathrm{d} \mathbf{r}^{\prime} \int_{0}^{1} \mathrm{~d} \lambda \operatorname{Tr}\left[\boldsymbol{\alpha}\left(\mathbf{r}, \mathbf{r}^{\prime}, \mathrm{i} u ; \lambda\right) \mathbf{T}^{\mathrm{int}}\left(\mathbf{r}, \mathbf{r}^{\prime}\right)\right]
$$

where $\mathbf{T}^{\text {int }}\left(\mathbf{r}, \mathbf{r}^{\prime}\right)$ is set to zero for $\mathbf{r}, \mathbf{r}^{\prime}$ from the same subsystem.

\subsection{Range Separation in Density Functional Theory}

The distinction between the short-range and long-range parts of the electron correlation energy becomes blurred in realistic systems at equilibrium geometries, where the overlaps between (or within) the interacting subsystems cannot be neglected. Some ab initio electronic-structure methods can treat both parts on an equal basis, but those are often computationally demanding and not applicable to large systems. It is therefore more common that the short-range and long-range parts are treated with different models, which must be somehow seamlessly joined, to avoid both omitting or double counting of some midrange part of the correlation. 
Notably, one of the most successful of such approaches is the combination of the density functional theory (DFT) in semilocal approximation for the short-range correlation and explicit vdW models for the long-range correlation.

The ACFD formula can be derived within the density functional for the total correlation energy of a general electronic system (see Callen and Welton 1951; Parr and Yang 1989, Sect. 8.6; Kohn et al. 1998), not only for the interaction energy of nonoverlapping bodies as in the previous section. In this form, it yields the exchange-correlation (XC) energy, $E_{\mathrm{xc}}$, the exchange part stemming from the antisymmetry of the electronic wave function,

$$
\begin{aligned}
& E_{\mathrm{xc}}=-\frac{1}{2 \pi} \int_{0}^{\infty} \mathrm{d} u \iint \mathrm{d} \mathbf{r} \mathrm{d} \mathbf{r}^{\prime} \int_{0}^{1} \mathrm{~d} \lambda \chi\left(\mathbf{r}, \mathbf{r}^{\prime}, \mathrm{i} u ; \lambda\right) v\left(\mathbf{r}, \mathbf{r}^{\prime}\right) \\
& =-\frac{1}{2 \pi} \int_{0}^{\infty} \mathrm{d} u \iint \mathrm{d} \mathbf{r} \mathrm{d} \mathbf{r}^{\prime} \int_{0}^{1} \mathrm{~d} \lambda \operatorname{Tr}\left[\alpha\left(\mathbf{r}, \mathbf{r}^{\prime}, \mathrm{i} u ; \lambda\right) \mathbf{T}\left(\mathbf{r}, \mathbf{r}^{\prime}\right)\right]
\end{aligned}
$$

Within time-dependent DFT, the true response functions can be further expressed in terms of the response functions of the KS noninteracting system, corresponding to $\lambda=0$, as a Dyson screening equation,

$$
\chi(\mathrm{i} u ; \lambda)=\chi(\mathrm{i} u ; 0)+\chi(\mathrm{i} u ; 0)\left(v+f_{\mathrm{xc}}(\mathrm{i} u ; \lambda) \chi(\mathrm{i} u ; \lambda)\right.
$$

where $f_{\mathrm{xc}}$ is the so-called $\mathrm{XC}$ kernel, in general an unknown system-dependent nonlocal function. In approximate vdW models, the XC effects contained in the kernel are usually incorporated implicitly into the effective polarizability,

$$
\boldsymbol{\alpha}(\mathrm{i} u ; \lambda) \approx \boldsymbol{\alpha}^{\mathrm{eff}}(\mathrm{i} u)+\boldsymbol{\alpha}^{\mathrm{eff}}(\mathrm{i} u) \mathbf{T} \boldsymbol{\alpha}(\mathrm{i} u ; \lambda)
$$

The XC energy can be formally divided into a short-range (sr) and long-range (lr) part by separating the double spatial integral in Eq. 12 into two parts using some range-separating function, $f$, which should decay at least exponentially fast for large distances,

$$
\begin{gathered}
\iint \mathrm{d} \mathbf{r}_{1} \mathrm{~d} \mathbf{r}_{2}=\iiint_{d} \mathbf{r}_{1} \mathrm{~d} \mathbf{r}_{2}\left(1-f\left(\mathbf{r}_{1}, \mathbf{r}_{2}\right)\right)+\iint \mathrm{d} \mathbf{r}_{1} \mathrm{~d} \mathbf{r}_{2} f\left(\mathbf{r}_{1}, \mathbf{r}_{2}\right) \\
\equiv \iint_{\mathrm{sr}} \mathrm{d} \mathbf{r}_{1} \mathrm{~d} \mathbf{r}_{2}+\iint_{1 \mathrm{lr}} \mathrm{d} \mathbf{r}_{1} \mathrm{~d} \mathbf{r}_{2}
\end{gathered}
$$

In practice, the short-range part is calculated via some semilocal $\mathrm{XC}$ functional, for which the corresponding $f$ is system-dependent and in general unknown. On the other hand, most vdW models have $f$ explicitly built in, and the aim is to find explicit $f$ for the vdW method that matches the implicit $f$ of a given $\mathrm{XC}$ functional. This is in general an unsolved problem, and most current approaches resort to a varying degree of empiricism.

The most radical difference in the range separation to semilocal DFT and vdW methods runs along the border between systems with uniform and nonuniform electron density. The vdW force between atomic bodies held together by covalent, ionic, or metallic binding is always caused by the long-range electron correlation, but not all effects of the long-range correlation are considered to be a vdW force. In 
metals, the electrons from the nonconducting bands are localized on atoms, which form nonuniform islands in the sea of approximately uniform electron density of the conducting electrons (Tao et al. 2010). Here, the long-range correlation between the conducting electrons contributes to the metallic binding. In nonmetals, however, all electrons are nonconducting, the electron density is nowhere uniform, and long-range correlation is mostly associated with vdW interactions. The electronic structure within a single uniform subsystem differs qualitatively in many aspects from that in a nonuniform system. In a uniform system, the exchange effects, the KS density response function, and the XC kernel decay only algebraically with distance (they are long-ranged) as a result of the conducting electrons, whereas they decay exponentially (they are short-ranged) in nonuniform systems (Ge and Lu 2015). (The true density response function decays algebraically in both cases because of electron correlation.) Correspondingly, semilocal and hybrid XC functionals capture both short-range and long-range part of the XC energy in uniform systems but only the short-range part in the nonuniform systems. The vdW interactions can be therefore associated with all long-range electron correlation except for that between conducting electrons within a single uniform subsystem, which is fortunately covered by semilocal and hybrid density functionals. The nonuniform situations include interactions between conducting electrons in disjoint metallic bodies; interactions of conducting electrons with localized electrons, either in the same metallic body or in other bodies; and all interactions between localized electrons.

The consequences of the differences between uniform and nonuniform systems for the range separation can be summarized as follows:

$$
\underset{\substack{E_{\mathrm{xc}} \\ \text { nonuniform : }}}{\text { uniform : }}=\overbrace{\underbrace{E_{\mathrm{x}}^{\mathrm{sr}}+E_{\mathrm{c}}^{\mathrm{sr}}}_{\text {semilocal/hybrid }}+\underbrace{E_{\mathrm{x}}^{\mathrm{lr}}}_{\approx 0}+\underbrace{E_{\mathrm{c}}^{\mathrm{lr}}}_{\mathrm{vdW}}}^{\text {semilocal/hybrid }}
$$

With the caveat about the uniform systems, the vdW interactions can then be associated with the long-range $\mathrm{XC}$ energy. In this setup, care must be taken about the potential double counting of the long-range XC energy in uniform systems from the semilocal or hybrid functionals and from the long-range ACFD formula. This double counting does not matter in situations when the result of a calculation is an energy difference, such as when calculating the adsorption energy of a molecule on a metal surface. But it may pose a problem in other cases, for instance, when investigating a lattice expansion of a metal.

\section{$5 \quad$ Classification of van der Waals Functionals}

Most existing models of long-range correlation can be described in terms of various approximations to the range-separated effective-polarizability version of the ACFD formula (Eqs. 12 and 14) (Hermann et al. 2017). One of them is the already 
discussed local representation of the effective polarizability. Two other general and common approximations are spatial coarse-graining of the system and truncation of the many-body expansion of the vdW energy.

\subsection{Coarse-Graining of Response Functions}

Given a set of functions, $w_{p}(\mathbf{r})$, that partition space into fragments, $\sum_{p} w_{p}(\mathbf{r}) \equiv 1$, and respective centers of the fragments, $\mathbf{R}_{p}$, each spatial function or operator, such as the dipole polarizability, can be represented as a sum over the partitioned components, $\boldsymbol{\alpha}_{p q}$, which can be in turn expanded in the basis of solid harmonics (multipole expansion), $\alpha_{p q, l^{\prime} m m^{\prime}}$, around the centers (Stone 2013),

$$
\boldsymbol{\alpha}\left(\mathbf{r}, \mathbf{r}^{\prime}, u\right)=\sum_{p q} w_{p}(\mathbf{r}) w_{q}\left(\mathbf{r}^{\prime}\right) \boldsymbol{\alpha}\left(\mathbf{r}, \mathbf{r}^{\prime} u\right) \equiv \sum_{p q} \boldsymbol{\alpha}_{p q}\left(\mathbf{r}, \mathbf{r}^{\prime}, u\right) \rightarrow \alpha_{p q, l l^{\prime} m m^{\prime}}
$$

(Here, $l, l^{\prime}$ start from 1, because the expanded quantity is a tensor. The corresponding expansion of the scalar density response function, $\chi$, would start from $l=l^{\prime}=0$.) The dipole potential is expanded correspondingly. Unlike the Fourier transformation, the multipole expansion is not invertible, but like the Fourier transformation, it introduces a correspondence between spatial integrals and infinite sums,

$$
\begin{aligned}
\mathbf{P}(\mathbf{r}, u) & =-\int \mathrm{d} \mathbf{r}^{\prime} \boldsymbol{\alpha}\left(\mathbf{r}, \mathbf{r}^{\prime}, u\right) \mathbf{E}\left(\mathbf{r}^{\prime}, u\right) \Longleftrightarrow \mathbf{P}_{p, l m}(u) \\
& =-\sum_{q, l^{\prime} m^{\prime}} \alpha_{p q, l l^{\prime} m m^{\prime}}(u) E_{q . l^{\prime} m^{\prime}}
\end{aligned}
$$

The motivation for this multipole reformulation is that because both $\mathbf{T}_{\mathrm{eff}}$ and $\mathbf{T}_{\mathrm{lr}}$ are long-ranged and their moments decay increasingly faster for higher l's; all the matrix multiplications (infinite sums) converge quickly and can be approximated well by finite sums.

The feasibility of the coarse-graining and multipole expansions is dictated by the choice of the fragments and the response properties of the system. In a nonuniform system, the nonlocal effective polarizability is exponentially localized on atoms, and atom-centered fragments are a natural basis of a quickly converging multipole expansion. In a uniform system, the effective polarizability is long-ranged, diffused, and there are no natural centers for the multipole expansion, leading to large higher moments and slow convergence or even divergence of the expansion. In principle, this problem is mitigated in combination with the KS-DFT, because the long-range XC energy within the uniform systems is captured by the semilocal or hybrid functional, and the multipole convergence of the correlation energy due to the interaction with a separate uniform or nonuniform system is helped by larger separations between the fragments. But such an interplay is not well understood, 
and none of the coarse-grained models discussed in this chapter take advantage of this cancellation.

\subsection{Truncation of Many-Body Expansion}

After inserting the Dyson screening equation into the ACFD formula, the couplingconstant integration can be carried out analytically either when using the effectivepolarizability formulation or by approximating the $\mathrm{XC}$ kernel with some form that depends on explicitly. In both cases, the $\lambda$-integration results in a logarithm expression such as

$$
E_{\mathrm{xc}} \approx-\frac{1}{2 \pi} \int_{0}^{\infty} \mathrm{d} u \iint \mathrm{d} \mathbf{r} \mathbf{r}^{\prime} \operatorname{Tr}\left[\ln \left(1-\alpha\left(\mathbf{r}, \mathbf{r}^{\prime}, \mathrm{i} u ; \lambda=0\right) \mathbf{T}\left(\mathbf{r}, \mathbf{r}^{\prime}\right)\right)\right]
$$

The operator logarithm is defined as an infinite series, and writing it out explicitly in terms of individual orders leads to a many-body decomposition of the $\mathrm{XC}$ energy,

$$
\begin{aligned}
& E_{\mathrm{xc}}=\frac{1}{2 \pi} \int_{0}^{\infty} \mathrm{d} u \iint \mathrm{d} \mathbf{r} \mathrm{d} \mathbf{r}^{\prime} \operatorname{Tr}\left[\boldsymbol{\alpha}\left(\mathbf{r}, \mathbf{r}^{\prime}, u ; 0\right) \mathbf{T}\left(\mathbf{r}, \mathbf{r}^{\prime}\right)\right] \\
& -\frac{1}{4 \pi} \int_{0}^{\infty} \mathrm{d} u \iiint \int \mathrm{d} \mathbf{r} \mathbf{r}^{\prime} \mathrm{d} \mathbf{r}^{\prime \prime} \mathrm{d} \mathbf{r}^{\prime \prime \prime} \operatorname{Tr}\left[\boldsymbol{\alpha}\left(\mathbf{r}, \mathbf{r}^{\prime}, u ; 0\right) \mathbf{T}\left(\mathbf{r}^{\prime}, \mathbf{r}^{\prime \prime}\right)\right. \\
& \left.\boldsymbol{\alpha}\left(\mathbf{r}^{\prime \prime}, \mathbf{r}^{\prime \prime \prime}, u ; 0\right) \mathbf{T}\left(\mathbf{r}^{\prime \prime \prime}, \mathbf{r}\right)\right]+\frac{1}{6 \pi} \cdots+\ldots
\end{aligned}
$$

The term "many-body" is best motivated in the coarse-grained models where the individual terms correspond to interactions between increasing number of fragments (bodies). (The order does not necessarily correspond to the number of bodies. At fourth order, for instance, some terms are a back-and-forth interaction between two bodies.) When constructed from the bare KS polarizability, the first order evaluates to the exact exchange, and the correlation energy starts at the second order. With any local approximation for the bare polarizability, the first term evaluates identically to zero, and the formula gives only the correlation energy. The long-range part of the second term is the leading term for vdW interactions and the basis of all nonlocal correlation functionals and coarse-grained pairwise methods discussed below. The third term corresponds to the Axilrod-Teller-Muto (ATM) three-body potential (Axilrod and Teller 1943; Mutō 1943) when coarse-grained to atoms.

\subsection{Random-Phase Approximation}

The approximations to the ACFD formula that are fully many-body and not coarsegrained can be based on the bare KS density response function. Because the KS density response function can be calculated directly from the KS orbitals via the 
so-called Adler-Wiser formula, these approximations are usually formulated and evaluated in the $\chi v$-representation rather than the $\alpha$ T-representation. Furthermore, because the KS response has a well-defined short-range structure, this construction allows the evaluation of the total XC energy, not only its long-range part, so the use of these methods goes beyond long-range correlation energy. Here, we discuss the methods from the perspective of $\mathrm{vdW}$ interactions.

The simplest of these methods is the RPA itself, which amounts to setting the XC kernel in Eq. 13 to zero (Ren et al. 2012). The omitted XC kernel is shortranged in nonuniform systems, but its omission influences both short-range and long-range correlation energy, because the short-range XC effects still influence the total polarizability of the system, which is manifested in the long-range correlation energy via the ACFD formula. As a result, although RPA does not suffer from any systematic errors in the long-range correlation energies, the overall accuracy is often worse than that of the many effective models discussed below (Olsen and Thygesen 2013b). This is further amplified in vdW systems in equilibrium geometries, where the short-range XC energy also contributes to the total interaction energy. Attempts at improvement go both ways, replacing the short-range correlation energy with a better model than RPA and improving the effective polarizability.

Kurth and Perdew (1999) suggested to correct the short-range correlation energy from RPA with that from a semilocal XC functional, in what they called the RPA+ method. Rather than explicitly range-separating the ACFD expression, RPA+ removes the RPA short-range part by subtracting correlation energy from a specially designed semilocal correlation functional, $E_{\mathrm{c}}^{\mathrm{GGA} @ \mathrm{RPA}}$, and reintroduces it back with a standard semilocal functional, $E_{\mathrm{c}}^{\mathrm{GGA}}$.

$$
E_{\mathrm{c}}^{\mathrm{RPA}+}=E_{\mathrm{c}}^{\mathrm{RPA}}-E_{\mathrm{c}}^{\mathrm{GGA} @ \mathrm{RPA}}+E_{\mathrm{c}}^{\mathrm{GGA}}
$$

$E_{\mathrm{c}}^{\mathrm{GGA} @ \mathrm{RPA}}$ is constructed in a similar way as standard functionals, but its uniform part is parameterized to reproduce the RPA energy of the electron gas rather than the true energy. RPA+ attempts to fix the short-range correlation energy of RPA, but the long-range part is unchanged, so the vdW force remains the same, and it is only the interaction due to electron-density overlap, which occurs at equilibrium, that can be possibly improved. Furthermore, the range separation in $\mathrm{RPA}+$ is unsystematic in the sense that there is no guarantee that $E_{\mathrm{c}}^{\mathrm{GGA} @ \mathrm{RPA}}$ and $E_{\mathrm{c}}^{\mathrm{GGA}}$ have the same effective range.

Toulouse et al. (2004) formulated a range-separated version of the KS scheme, in which the $\mathrm{XC}$ functional is designed from the beginning to treat only the shortrange part of the electron correlation. This leads to an alternative range separation of the ACFD formula, in which $\alpha(\lambda)$ is not the polarizability of the wave function that minimizes $\langle\Psi|\widehat{T}+\lambda \widehat{V}| \Psi\rangle$ but rather of one that minimizes $\left\langle\Psi\left|\widehat{T}+\lambda \widehat{V}_{\text {lr }}\right| \Psi\right\rangle$ (Toulouse et al. 2009). In this scheme, the RPA of the Dyson-like equation results in a model in which the effective polarizability is still equal to the bare KS polarizability, like in normal RPA, but the effective dipole operator is only the long-range part of the full operator. The underlying assumption then is that the dipole operator and the XC kernel partially cancel out at short range, giving a 
different estimate of the effective polarizability than normal RPA. This is supported by numerical evidence on select small systems. A similar scheme, proposed earlier by Kohn et al. (1998), also uses a range-separated version of the KS scheme, but instead of obtaining the true polarizability at the RPA level, $\chi(\lambda)$ is obtained for each $\lambda$ by explicitly perturbing the corresponding $\lambda$-scaled system with electric field.

A straightforward way to improve the RPA is to devise approximate XC kernels, which improves the short-range behavior of the polarizability and hence both shortrange and long-range correlation energies. Extending the LDA to the time domain, the adiabatic LDA (ALDA) assumes that the XC kernel has no memory, leading to a frequency-independent local XC kernel. Unlike LDA, which is exact for the uniform electron gas (UEG), ALDA does not give the true XC kernel of the UEG (which is nonlocal in both time and space) and violates several known properties of the true XC kernel. Despite that, it is a useful approximation in TD-DFT calculations when one is interested only in a certain range of the frequency spectra. Still, it turns out not to be a good approximation in the ACFD formula, where it gives worse results than the absent XC kernel of the RPA (Lein et al. 2000).

Olsen and Thygesen (2012) constructed a correction to ALDA by fixing its largeq (short-range) behavior in the UEG to better reproduce the known exact behavior. Taking this renormalized ALDA (rALDA) kernel, transforming back to real space, and using the mean density in two points as the corresponding uniform density, this procedure gives a universal XC kernel. This construction is computationally no more demanding than RPA but improves upon RPA in almost every case tested (Olsen and Thygesen 2013a, 2014). The rALDA XC kernel gives a more realistic short-range screening of the bare KS polarizability, resulting in more accurate longrange correlation energies and better description of $\mathrm{vdW}$ systems.

A different path toward improving the accuracy of RPA can be taken using the many-body perturbation (MBPT) theory. This is possible because, as GellMann and Brueckner (1957) showed, yet another equivalent definition of RPA is via a certain subset of Feynman diagrams, the so-called ring diagrams. Summing different subsets of the diagrams similar to those corresponding to RPA then leads to different RPA-like models and sometimes confusing terminology, when a certain modification of the XC kernel in RPA is equivalent to adding additional terms to the RPA XC energy that do not seem to be related to RPA (Scuseria et al. 2008; Jansen et al. 2010; Ángyán et al. 2011).

The second-order Møller-Plesset correlation energy (MP2) consists of the Coulomb direct and exchange terms, of which only the former is long-ranged. In this context, RPA can be understood as the sum of all MP2-like direct terms (ring diagrams) in the infinite MBPT expansion. Similarly, the MP2 exchange can be renormalized by replacing one of the Coulomb interactions with the RPA sequence of ring diagrams, leading to the second-order screened exchange (SOSEX). Furthermore, unlike in the Møller-Plesset perturbation theory, where the first order is guaranteed to be zero, single-electron excitations contribute to the XC energy in the MBPT based on KS orbitals. Combining RPA, SOSEX, and RPA-renormalized single-excitation correction then results in the renormalized second-order perturbation theory (rPT2) (Ren et al. 2011, 2013). Although the 
MP2 exchange term is short-ranged, the renormalization in SOSEX is long-ranged, and the long-range correlation energy of rPT2 is different from that of RPA. The combined improvements of the short-range and long-range XC energy in rPT2 compared to RPA lead to improved accuracy in vdW binding energies.

\subsection{Nonlocal van der Waals Density Functionals}

The models of long-range correlation energy discussed in this section are in the class of approximations to the ACFD formula that truncate the many-body expansion at second order, but do not do any spatial coarse-graining. This leads to XC functionals that are characterized by nonlocal dependence of the XC energy density on the electron density via some nonlocal kernel, $\Phi$,

$$
\begin{aligned}
E_{\mathrm{c}}^{\mathrm{nl}-\mathrm{df}} & =-\frac{1}{2} \int \mathrm{d} \mathbf{r} \mathrm{d} \mathbf{r}^{\prime} n(\mathbf{r}) n\left(\mathbf{r}^{\prime}\right) \Phi[n]\left(\mathbf{r}, \mathbf{r}^{\prime}\right) \\
& =-\int \mathrm{d} \mathbf{r} n(\mathbf{r}) \int \mathrm{d} \mathbf{r}^{\prime} \frac{1}{2} n\left(\mathbf{r}^{\prime}\right) \Phi[n]\left(\mathbf{r}, \mathbf{r}^{\prime}\right)
\end{aligned}
$$

The effective polarizability is approximated with a local isotropic polarizability,

$$
\boldsymbol{\alpha}_{\mathrm{eff}}\left(\mathbf{r}, \mathbf{r}^{\prime}, u\right) \approx \mathbf{I} \alpha_{\mathrm{eff}}(\mathbf{r}, u) \delta\left(\mathbf{r}-\mathbf{r}^{\prime}\right)
$$

This results in the first-order term in the many-body expansion being zero, which means that such a functional cannot capture any exchange energy, which is intentional, since the nonlocal functionals are designed to capture only the longrange correlation energy. The locality of the effective polarizabilities reduces two of the four integrals in the second-order term, and the isotropy allows to take the polarizabilities out of the trace in Eq. 20.

A general form of the local effective polarizability used in many models is obtained from the polarizability of a harmonic oscillator by setting the ratio of the charge and mass to that of an electron, $q / m=1$, and substituting the electron density for the charge,

$$
\alpha_{\mathrm{tot}}^{\mathrm{HO}}(\mathrm{i} u)=\frac{q^{2} / m}{\omega^{2}+u^{2}} \rightarrow \alpha_{\mathrm{eff}}[n](\mathbf{r}, \mathrm{i} u)=\frac{n(\mathbf{r})}{\omega_{\mathrm{eff}}^{2}[n](\mathbf{r})+u^{2}}
$$

Besides the obvious reason of modelling electrons, the charge-mass ratio of one is motivated by the $f$-sum rule for an electronic system that dictates that $\alpha_{\text {tot }}(\mathrm{i} u) \rightarrow N / u^{2}$ ( $N$ is the number of electrons), which the form above automatically satisfies. (Strictly speaking, this is not necessary, because the rule does not need to be satisfied in any local form, and furthermore, the local effective polarizability is not supposed to integrate to the total polarizability without any long-range coupling. However, the local form is a straightforward way to satisfy the global rule.) The local

effective resonance frequency, $\omega_{\text {eff }}^{2}$, can be in general any functional of the electron density but is often approximated semilocally. 
The approximated ACFD formula can then be recast in the form of a nonlocal density functional, where the nonlocal kernel is a functional of the effective resonance frequency and some (so far unspecified) range-separating function,

$$
\begin{aligned}
E_{\mathrm{c}, \mathrm{lr}} & \approx-\frac{1}{2} \iint \mathrm{d} \mathbf{r d} \mathbf{r}^{\prime} n(\mathbf{r}) n\left(\mathbf{r}^{\prime}\right) \frac{3}{\pi} \int_{0}^{\infty} \mathrm{d} u \\
& \frac{1}{\omega_{\mathrm{eff}}^{2}[n](\mathbf{r})+u^{2}} \frac{1}{\omega_{\mathrm{eff}}^{2}[n]\left(\mathbf{r}^{\prime}\right)+u^{2}} \frac{f\left(\mathbf{r}, \mathbf{r}^{\prime}\right)}{\left|\mathbf{r}-\mathbf{r}^{\prime}\right|^{6}}
\end{aligned}
$$

The asymptotic behavior of the long-range correlation energy calculated in this way is fully specified by $\omega_{\text {eff. }}$.

The first general functional of this form, referred to simply as the vdW density functional (vdW-DF), was developed by Dion et al. (2004). Although the derivation of vdW-DF starts from the ACFD formula, it follows quite a different direction than the framework in this chapter, and most of the approximations along the way are done in reciprocal space, until everything is transformed back to real space in the end. However, the final result can still be cast in the form of Eq. 25. The effective resonance frequency in the vdW-DF is constructed from a GGA-type XC energy density. The first equality is motivated by using $\omega_{\text {eff }}^{2}$ to calculate the $\mathrm{XC}$ energy of a slowly varying electron gas via the ACFD formula. The particular choice of the semilocal approximation to the XC energy density is rather arbitrary and completely independent of the semilocal functional potentially used to complete the vdW-DF at short range.

A serious disadvantage of the vdW-DF in light of other long-range correlation models is that its range-separating function is fixed by the underlying theory. Because of the construction in the reciprocal space, the parameter $A$ appears both in the effective resonance frequency and the range-separating function. Since the asymptotic behavior of any nonlocal functional depends only on $\omega_{\text {eff }}$, not the rangeseparating function, the parameter $A$ is essentially fixed, and there is no remaining freedom in the range-separating function that could be adjusted for a particular choice of a short-range semilocal functional in a full KS-DFT calculation.

The form of the range-separating function is complex due to the reciprocal-space formulation, but there are two underlying physical motivations for it. When the two oscillators given by the resonance frequencies $\omega_{\text {eff }}$ are close to each other such that their ground-state wave functions would overlap, the underlying model does not work anymore, the corresponding part of the XC energy must be covered by the semilocal functional, and the dipole coupling must be damped. This is effectively achieved by increasing the resonance frequency as $k^{2}$ in the reciprocal space. The second damping mechanism is that the nonlocal functional must evaluate to zero for the uniform electron gas, whose long-range correlation energy is already covered by a semilocal or a hybrid functional. This forces the range-separating function to negative values at short range, to counterbalance the attractive contribution from the long range. 
The complex form of vdW-DF was simplified in the VV09 functional, which used a substantially different form of $\omega_{\text {eff }}$,

$$
\omega_{\mathrm{VV}}^{2}[n](\mathbf{r})=\frac{4 \pi}{3} n(\mathbf{r})+C \frac{|\nabla n|^{4}}{n^{4}}
$$

Here, $4 \pi n$ is the resonance frequency of the macroscopic (small-q limit) plasmon fluctuations of the uniform electron gas. The factor of $1 / 3$ comes from the ClausiusMossotti relation between the microscopic local polarizability and the macroscopic dielectric function. The density-gradient term is a local model of a bandgap obtained from considering the behavior of the electron density in the density tail of a finite system. The range-separating mechanism of VV09 is still constructed in reciprocal space. The latest attempt at a simplified formulation of the vdW-DF, named VV10, was constructed entirely in real space (Vydrov and Van Voorhis 2010). Both the resonance frequency and range-separating function of Eq. 25 have a simple form in VV10. The former is the same as in VV09, and the latter is constructed using the same mechanism of reduced polarizabilities of overlapped oscillators as in the original vdW-DF but in real space.

\subsection{Pairwise Interatomic Models}

The oldest approaches to fix the missing long-range electron correlation in HF or semilocal KS-DFT calculations are of the interatomic pairwise form,

$$
E_{\mathrm{c}, \mathrm{lr}} \approx-\frac{1}{2} \sum_{p q} C_{6, p q} \frac{f\left(\mathbf{R}_{p}, \mathbf{R}_{q}\right)}{\left|\mathbf{R}_{p}-\mathbf{R}_{q}\right|^{6}}
$$

Here, $f$ is some range-separating (damping) function, $\mathbf{R}_{q}$ are the atom coordinates, and the so-called dispersion coefficients, $C_{6, \mathrm{pq}}$, determine the asymptotic interaction between two atoms. This type of interatomic potential has origin in empirical force fields dating back to the Lennard-Jones potential, even before it was clear that the correct leading term of the vdW force is $1 / R^{6}$. In the context of electronic-structure methods, it was first used by Hepburn et al. (1975) to correct interaction curves of rare-gas dimers from HF calculations. This approach was later extended to molecules and KS-DFT calculations, and the $C_{6}$ coefficients were extended to a wider range of systems (Halgren 1992; Mooij et al. 1999; Elstner et al. 2001; Wu and Yang 2002). Grimme (2004) then presented a parametrization of $C_{6}$ and $f$, termed DFT-D ("D" for dispersion), that could in principle be applied to any molecule or solid, in combination with any $\mathrm{XC}$ functional. This marked a start of routine addition of the long-range correlation energy to semilocal KS-DFT calculations.

The pairwise interatomic model of Eq. 27 can be obtained as a coarse-grained truncated approximation to the ACFD formula. The derivation follows the same 
course of second-order truncation and local approximation to the effective polarizability as nonlocal vdW density functionals but starting from the coarse-grained multipole-expanded ACFD formula (see Eq. 18),

$$
E_{\mathrm{c}, \mathrm{lr}} \approx-\frac{1}{4 \pi} \int_{0}^{\infty} \mathrm{d} u \operatorname{Tr}_{p, l m}\left(\boldsymbol{\alpha}_{\mathrm{eff}}(\mathrm{i} u) \mathbf{T}_{\mathrm{lr}} \boldsymbol{\alpha}_{\mathrm{eff}}(\mathrm{i} u) \mathbf{T}_{\mathrm{lr}}\right)
$$

Here, the trace is over multipole moments and fragments, which are chosen to be atoms in most cases. (In this context, the formal definition of an atom in a molecule is given by some partition function.) Approximating the local effective polarizability as isotropic, $\alpha_{\text {eff, } p l l^{\prime} m m^{\prime}}=\delta_{l l^{\prime}} \delta_{m m^{\prime}} \alpha_{\mathrm{eff}, p l}$, the formula is reduced as in the case of nonlocal vdW XC functionals. The standard pairwise formula of Eq. 27 is recovered by keeping only the lowest dipole-dipole term $\left(l=l^{\prime}=1, K_{11}=6\right)$, where the expression for the corresponding dispersion coefficient is called the Casimir-Polder integral,

$$
\left.C_{6, p q}=\frac{3}{\pi} \int_{0}^{\infty} \mathrm{d} u \alpha_{\mathrm{eff}, p 1}(\mathrm{i} u) \alpha_{\mathrm{eff}, q 1} \text { (i } u\right)
$$

Some pairwise methods are formulated directly in terms of the dispersion coefficients, not the underlying polarizabilities, in which case approximate combination rules for calculating unknown heteronuclear coefficients from known homonuclear coefficients are useful. Such rules can be derived from the Casimir-Polder integral using some model polarizability. An often used rule is obtained from the harmonicoscillator model,

$$
C_{6, p q}^{\mathrm{HO}}=\frac{3}{\pi} \int_{0}^{\infty} \mathrm{d} u \frac{\alpha_{p}(0) \omega_{p}^{2}}{\omega_{p}^{2}+u^{2}} \frac{\alpha_{q}(0) \omega_{q}^{2}}{\omega_{q}^{2}+u^{2}}=\frac{2 C_{6, p p} C_{6, q q}}{C_{6, p p} \frac{\alpha_{q}(0)}{\alpha_{p}(0)}+C_{6, q q} \frac{\alpha_{p}(0)}{\alpha_{q}(0)}}
$$

Using the single-pole polarizability of the harmonic oscillator in situations where the true spectrum is more complex, such as in the equation above, is called the Unsöld (1927) approximation.

The models of Grimme, mentioned above, are different from the rest discussed in this section in that they are formulated only in terms of the geometry of a molecule, $\{\mathbf{R} p\}$, not the electron density. This makes them straightforwardly useful even for empirical short-range electronic models that do not produce any electronic density, but at the same time, it makes it much harder to achieve truly general models, because the electron density encodes much useful information about the system.

The first version of DFT-D used fixed homonuclear $C_{6}$ coefficients, the combination of Eq. 30 with all polarizability ratios set to 1 and a range-separating function constructed from vdW radii that did not go to 1 in infinity (Grimme 2004). The second version was a numerical reparametrization of the first one with a changed combination rule, which set the polarizability ratios equal to those of the $C_{6}$ coefficients (Grimme 2006). In the first and second version, the atomic 
$C_{6}$ coefficients do not depend on the molecular environment, which is a crude approximation. The third version was an improvement in several regards (Grimme et al. 2010). The range separation was modified to obey the correct asymptotic behavior. An elementary dependence of the $C_{6}$ coefficients on the environment was included via geometrical factors estimating the coordination number of an atom. The dipole-quadrupole term $\left(l=1, l^{\prime}=2\right)$ was included, and a three-atom correction was suggested, which is the third-order triple-dipole term in the logarithm expansion of the coarse-grained ACFD formula. The corresponding dispersion coefficients, $C_{8}$ and $C_{9}$, are obtained by combination rules similar to those for the $C_{6}$ coefficient.

Soon after the first version of DFT-D and in stark contrast to it, Becke and Johnson (2005b) developed a method to calculate $C_{6}$ coefficients from first principles, using an approximation to the polarizability based on the dipole moment of the XC hole of the HF model, the exchange-hole dipole method (XDM). Their initial derivation was rather heuristic, with a wrong prefactor, but the final result can be in fact obtained directly from the Casimir-Polder integral using the fluctuationdissipation theorem for the density response function and the Unsöld approximation (Ángyán 2007; Heßelmann 2009; Ayers 2009). A semilocal approximation to the XC hole by Becke and Roussel (1989) works as well as that from the HF model and with the additional benefit of reduced computational complexity (Becke and Johnson 2005a). To formulate a general interatomic pairwise method, the (local) dipole moment of the $\mathrm{XC}$ hole is coarse-grained using the partitioning scheme devised by Hirshfeld (1977). In this scheme, the atomic partition functions, $w_{p}$, are constructed from radially averaged electron densities of isolated atoms, $n^{\text {free }}$,

$$
w_{p}^{\text {Hirsh }}(\mathbf{r})=\frac{n_{p}^{\text {free }}\left(\left|\mathbf{r}-\mathbf{R}_{p}\right|\right)}{\sum_{q} n_{q}^{\text {free }}\left(\left|\mathbf{r}-\mathbf{R}_{q}\right|\right)}
$$

The corresponding static dipole polarizabilities of the atomic fragments are calculated from free-atom dipole polarizabilities, assuming that they scale linearly with the Hirshfeld measure of a volume (Hirshfeld volume),

$$
\begin{gathered}
\alpha_{p 1}(0)=\alpha_{p 1}^{\text {free }}(0) \frac{V_{p}^{\text {Hirsh }}[n]}{V_{p}^{\text {Hirsh }}\left[n^{\text {free }}\right]} \\
V_{p}^{\text {Hirsh }}[n]=\int \mathrm{d} \mathbf{r} n(\mathbf{r}) w_{p}^{\text {Hirsh }}(\mathbf{r})\left|\mathbf{r}-\mathbf{R}_{p}\right|^{3}
\end{gathered}
$$

The fragment $C_{6}$ coefficients are then calculated from the fragment polarizabilities and coarse-grained XC hole dipole moment. The harmonic-oscillator combination rule is used to get the rest of the $C_{6}$ coefficients. The XDM can be extended to higher multipole dispersion coefficients by calculating higher multipole moments of the XC hole polarization around each atomic center (Becke and Johnson 2006; Johnson and Becke 2006). 
A simple yet accurate interatomic pairwise method was developed by Tkatchenko and Scheffler (2009) (TS), who extended the free-atom scaling approach to all the atomic parameters, including the $C_{6}$ coefficients and the vdW radii, and thus formulated the calculation of interatomic pairwise vdW interactions into a true density functional. Assuming that the excitation energies of the atoms are independent of the volume, the Unsöld approximation and the Casimir-Polder integral dictate that the $C_{6}$ coefficients scale with the second power of the Hirshfeld volume ratio,

$$
C_{6, p q}=C_{6, p q}^{\mathrm{free}}\left(\frac{V_{p}^{\mathrm{Hirsh}}[n]}{V_{p}^{\mathrm{Hirsh}}\left[n^{\mathrm{free}}\right]}\right)^{2}
$$

The free-atom reference values may not be the most effective choice in metals and some solids, whose electron density is often substantially different from the superposition of free-atom densities. Zhang et al. (2011) and Ruiz et al. (2012) used an adapted TS method, where the reference values are obtained from bulk macroscopic dielectric constant.

Sato and Nakai $(2009,2010)$ developed an atomic pairwise method based on the local effective polarizability functional from the vdW-DF-09 nonlocal functional. A system is described by the local effective polarizability given by the harmonicoscillator formula with the resonance frequency from Eq. 26. The atomic fragments are defined using the partitioning functions from the scheme by Becke (1988), which is most often used to define atomic radial grids in KS-DFT calculations, but here it is used as an alternative to the Hirshfeld partitioning. The partitioned polarizability is used to calculate a coarse-grained representation of the system via multipole expansion and Casimir-Polder integrals up to the $C_{10}$ coefficient.

Silvestrelli (2008) formulated a pairwise method in which the coarse-grained fragments are not atoms but Wannier functions (WFs) (Marzari et al. 2012). Wannier functions are any set of localized one-electron wave functions that in principle form a complete basis. In finite molecular systems, they are called Boys orbitals. The Wannier functions of conducting and nonconducting electrons are localized algebraically and exponentially, respectively. In the vdW-WF method, each WF is approximated with a single spherically symmetric exponential function that has the same width (second central moment) as the true WF. The polarizability of the approximate WF is calculated with the polarizability functional of Andersson et al. (1996) (ALL). Here, $n_{p}$ is the electron density of the WF and $k$ is a nonempirical constant. The $C_{6}$ coefficients between the WFs are calculated from the CasimirPolder integral, and the range-separating function is the same as in the TS method, with vdW radii of the WFs defined via an electron density cutoff (Silvestrelli et al. 2009). The vdW-WF scheme has two theoretical shortcomings: first, the partitioning of the total electron density is only approximate because of the use of the approximate WFs, and second, the ALL polarizability functional was designed for the total electron density, not one-electron densities. 
Coarse-grained methods in which the fragment polarizabilities and $C_{6}$ coefficients are calculated directly, rather than obtained by explicit partitioning of some continuous quantity, may be sensitive to a particular choice of the partitioning scheme. This motivated a series of modified Hirshfeld partitioning schemes that should capture better the redistribution of the electron density in a molecule with respect to free atoms. Steinmann and Corminboeuf $(2010,2011)$ adapted the XDM to use the self-consistent Hirshfeld scheme, which gives a more consistent description of ionic systems (Bultinck et al. 2007). Bučko et al. $(2013,2014)$ did the same with the TS method. The self-consistent Hirshfeld partitioning uses the same stockholder formula in Eq. 31 as the original scheme, but the reference densities are generalized and depend recursively on the partitioning, leading to equations that need to be solved iteratively (Verstraelen et al. 2012). A common form of the generalized reference densities, used in the modified XDM and TS methods, is a linear combination of free-atom and free-ion densities that maintains the charge of the Hirshfeld-partitioned atomic density. This scheme is complicated by the instability of many isolated anions, which requires addition of auxiliary negative charges, making the partitioning somewhat arbitrary.

\subsection{Many-Body Dispersion}

The fourth class of approximations to the ACFD formula covers nontruncated coarse-grained models. A common theme of all such models is to interpret the Unsöld approximation with its single resonance frequency literally and model a real molecular system as a collection of coupled charged oscillators. The corresponding Hamiltonian describes a system of distinguishable particles characterized by a charge, $q_{i}$, and a mass, $m_{i}$, each having its own harmonic potential defined by the resonance frequency, $i$, and a center, $\mathbf{R}_{i}$, interacting via the Coulomb force,

$$
\begin{aligned}
\widehat{H}_{\mathrm{osc}} & =\sum_{i} \frac{\widehat{\mathbf{p}}_{i}^{2}}{2}+\sum_{i} \frac{1}{2} m_{i} \omega_{i}^{2}\left|\widehat{\mathbf{r}}_{i}-\mathbf{R}_{i}\right|^{2} \\
& +\sum_{i<j} q_{i} q_{j}\left(\frac{1}{\left|\widehat{\mathbf{r}}_{i}-\widehat{\mathbf{r}}_{j}\right|}-\frac{1}{\left|\widehat{\mathbf{r}}_{i}-\mathbf{R}_{j}\right|}-\frac{1}{\left|\mathbf{R}_{i}-\widehat{\mathbf{r}}_{j}\right|}+\frac{1}{\left|\mathbf{R}_{i}-\mathbf{R}_{j}\right|}\right)
\end{aligned}
$$

The centers of the harmonic potentials additionally host a compensating charge of the opposite sign. If the centers are the same as those of the atoms, this Hamiltonian can be interpreted as a very crude approximation to the electronic Hamiltonian, in which all electrons of individual atoms are described by distinguishable psuedoelectrons that move in an effective potential which is the combined result of the nuclear potential and the mean field of the electrons. In particular, any exchange effects and hence charge transfer and delocalization are not considered. Expanding the Coulomb operator in a multipole series and keeping only the dipole term result in dipole-coupled oscillator Hamiltonian, 


$$
\begin{aligned}
\widehat{H}_{\mathrm{dosc}} & =\sum_{i} \frac{\widehat{\mathbf{p}}_{i}^{2}}{2}+\sum_{i} \frac{1}{2} m_{i} \omega_{i}^{2}\left|\widehat{\mathbf{r}}_{i}-\mathbf{R}_{i}\right|^{2} \\
& +\sum_{i<j} q_{i} q_{j}\left(\widehat{\mathbf{r}}_{i}-\mathbf{R}_{i}\right) \mathbf{T}\left(\mathbf{R}_{j}-\mathbf{R}_{i}\right)\left(\widehat{\mathbf{r}}_{j}-\mathbf{R}_{j}\right)
\end{aligned}
$$

A useful property of this Hamiltonian is that it can be solved exactly by coordinate transformation into a system of uncoupled quasi-oscillators, which describe different collective oscillations. The ground-state wave function of such a system is then a simple product of the single-oscillator ground-state wave functions, and the ground-state energy is a sum of the single-oscillator ground-state energies, $E_{0}=\sum_{n} \tilde{\omega}_{n} / 2$. Drawing analogy with the RPA, the individual oscillators model the particle-like quasi-electrons in some coarse-grained way, while the coupled oscillations model the wavelike electron oscillations. This Hamiltonian has been used many times to obtain various qualitative properties of long-range electron correlation (Bade 1957; Bade and Kirkwood 1957; Mahan 1965; Lucas 1967; Renne and Nijboer 1967; Donchev 2006) but only recently to formulate general quantitative methods.

The relevance of the dipole-coupled oscillator model to the true electronic system can be derived directly from the coarse-grained ACFD formula by performing the frequency integration analytically (Tkatchenko et al. 2013). When truncated at the dipole term, the approximate long-range correlation energy is then equal to the difference in the ground-state energy between the dipole-coupled oscillators and noninteracting oscillators. The exact equivalence between the dipole-coupled oscillators and the approximated ACFD formula breaks when going beyond the dipole approximation. The effective Hamiltonian derived from the ACFD formula is always bilinear in the interaction and contains one oscillator per each fluctuating moment (dipole, quadrupole, etc.). In contrast, the coupled-oscillator Hamiltonian has always $3 N$ coordinates, independent of the degree of the multipole expansion of the Coulomb operator, and the interaction terms above the dipole order are formed from nonlinear combinations of the coordinates, making the Hamiltonian unsolvable in closed form.

The use of the coupled-dipole approach to formulate general methods for the long-range correlation energy was initiated in the many-body dispersion (MBD) model developed by Tkatchenko et al. (2012). MBD reuses the effective dynamic polarizability as approximated in the TS pairwise method and combines it with a physically motivated effective dipole operator. Motivated by the Gaussian shape of the harmonic-oscillator ground-state wave function, the dipole potential in MBD, $\mathbf{T}_{\mathrm{gg}}$, is derived from the screened Coulomb interaction between two Gaussian unitcharge densities (Mayer 2007), with widths derived from the corresponding dipole polarizabilities. In general, the dipole potential in the Dyson equation should be different from that in the ACFD formula and independent of the XC functional used for the short-range part of the correlation energy. To circumvent this obstacle, Ambrosetti et al. (2014b) separated $\mathbf{T}_{\mathrm{gg}}$ into the long-range part and the short-range 
remainder. The long-range correlation energy is then calculated in two steps. First, the effective polarizability is screened by the short-range dipole potential via the Dyson equation. Second, the dipole-coupled Hamiltonian is solved with the longrange dipole potential.

Silvestrelli (2013) developed another method inspired by MBD in which the oscillators do not model the response of the atoms but of Wannier functions. This Wannier-based MBD is to the pairwise vdW-WN method as what the rangeseparated MBD is to the pairwise TS method. Unlike in vdW-WN, here the polarizabilities of the Wannier functions are not calculated using a local polarizability functional but directly from the Hirshfeld volumes of the Wannier functions.

\section{$6 \quad$ Applicability in Material Modelling}

Most vdW models have been designed following some set of theoretical principles (as is common in DFT), rather than obtained by a straightforward application of systematic approximations (as is common in quantum chemistry). As a result, a careful attention must be paid to the evaluation of the accuracy of the models, to avoid any systematic bias, both within and between different classes of systems, and to know the level of uncertainty in predicted quantities that one may expect. In this regard, the systematic verification of a given DFT $+\mathrm{vdW}$ method is usually achieved through comparison against the results of higher-level (more costly and more accurate) theoretical methods or experimental results with sufficient resolution.

In the case of organic molecules and materials, several benchmark sets of binding energies of complexes and lattice energies of molecular crystals have been established that allow for systematic testing of vdW models. For smaller molecules, the reference data have been obtained by high-level correlated methods of quantum chemistry (Jurečka et al. 2006; Řezáč et al. 2011), for larger molecules by diffusion quantum Monte Carlo (Ambrosetti et al. 2014a) and for molecular crystals by extrapolating experimental lattice enthalpies to zero temperature (Otero-de-la-Roza and Johnson 2012; Reilly and Tkatchenko 2013b). Initially, the development of $\mathrm{vdW}$ models was largely driven by their performance for small molecules on the S22 and S66 benchmark sets, and currently most popular DFT+vdW methods are able to achieve accuracies of $10-20 \mathrm{meV}$ (better than 10\%). The remaining errors are due to inaccuracy in the asymptotic vdW coefficients, empirical parameters in damping functions, and errors in the XC functional. Because of such rather uniform performance of different methods for small molecules, the focus has shifted to assessing the performance for larger systems. Here, in fact, the differences are more prominent, because the vdW energy makes a much larger relative contribution to cohesion. For example, for polarizable supramolecular systems, such as the "buckyball catcher" complex, pairwise dispersion corrections overestimate the binding energy by $0.4-0.6 \mathrm{eV}$, whereas including many-body dispersion effects reduces this error to $0.1 \mathrm{eV}$.

For periodic molecular crystals, some coarse-grained DFT $+\mathrm{vdW}$ methods are able to achieve remarkable accuracy of $40-50 \mathrm{meV}$ per molecule (5\%), compared 
to experimental results (Reilly and Tkatchenko 2013a, b; Brandenburg and Grimme 2014). Since the difference in lattice energies between various available experiments is on the same order of magnitude, this highlights the mature state of vdW dispersion corrections to semilocal DFT. The nonlocal density functionals yield somewhat larger errors (Otero-de-la-Roza and Johnson 2012). Understanding the performance of different vdW-inclusive methods for large molecular systems is still a subject of ongoing research. Accurate description of vdW interactions becomes even more relevant for the relative energetics of molecular systems, which are essential to predict the correct polymorphic behavior of molecular crystals (Reilly et al. 2016).

Beyond organic materials, the performance of DFT $+\mathrm{vdW}$ methods have started to be tested only recently. Reference lattice and interlayer binding energies calculated with RPA exist for a range of bulk solids (Harl et al. 2010; Schimka et al. 2013) and layered materials (Björkman et al. 2012; Björkman 2012, 2014), but the accuracy of RPA on the organic systems is comparable to that of DFT+vdW methods. Alternatively, experimental data are available for many of these systems. Of the different material types, the performance of individual methods for semiconductors is usually comparable to that of the organic compounds. In contrast, the accuracy with which ionic solids and metallic materials are described differs greatly between vdW models. In some cases, this has motivated the development of different flavors of vdW models, each targeting a specific class of materials.

\section{$7 \quad$ Toward the Ultimate van der Waals Model}

Despite the numerous advances in recent years discussed in the previous sections, a general, accurate, and efficient vdW model is not yet available. Arguably, this is a result of each of the current vdW models having some theoretical deficiencies, some of which are shared by all the models. Therefore, and to stimulate the reader with potential research problems, we conclude this chapter with a list of theoretical features that are missing from some or all current vdW models. Furthermore, we discuss which types of materials do we expect to benefit from potential addition of such features.

- All current vdW models assume a localized noninteracting polarizability, which is appropriate for gapped electronic systems, but not for gapless ones. At the same time, it is not clear what is the importance of the delocalized part of the response to vdW interactions in different circumstances. For instance, one can expect that the delocalized fluctuations will be manifested more strongly in the case of two metallic objects and less in the case of a molecule adsorbed on a metallic surface. Developing a unified model that uses both the localized and delocalized parts of the electronic response would enable treating the widest possible range of systems from purely covalent to purely metallic.

- Most current interatomic approaches assume a single-oscillator frequency dependence of the polarizability. This may be adequate for lighter elements of the periodic table in which majority of the response comes from the valence 
electrons with similar response properties. However, the core shells contribute nonnegligibly to the polarizability of heavier elements, and in such cases the use of two oscillators to capture separately the response of inner-shell and valence electrons could be beneficial to accuracy.

- All current models of effective polarizability assume isotropic local response. Could the directionality of the density gradient be used to construct an anisotropic polarizability model? The part of anisotropy resulting from the long-range interactions (for instance, due to specific packing in a molecular crystal) is already captured in many-body vdW effects. But the part stemming from short-range interactions between neighboring atoms (such as in planar aromatic compounds) is currently neglected.

- The VV functional is perhaps the most accurate semilocal functional for the local polarizability, but it is still lacking in accuracy to some of the interatomic approaches. Could this be potentially remedied by including dependence on the kinetic energy density or the second derivative of the electron density? Are there some exact constraints and limits on the polarizability functional? Improvements of this type can be expected to improve the accuracy of the vdW models across the whole spectrum of materials.

- All methods that use vdW models are based on some empirical coupling of the short-range and long-range parts. The empiricism necessarily hinders generality and introduces bias toward the system on which the coupling was constructed. Many theoretical results are available about general properties of XC functionals yet none so far that would enable a more rigorous coupling between semilocal DFT and vdW models. This question is especially relevant in systems where long-range $\mathrm{vdW}$ interactions contribute only partially to the total interaction energy, such as smaller organic complexes or organic/inorganic systems.

- Except for the costly RPA-based methods, there is no general vdW model that would be simultaneously many-body while treating also higher multipole moments of the polarizability. Some pairwise interatomic models include higher multipole moments, and nonlocal vdW functionals do not need to because of the lack of coarse-graining, but none of them are fully many-body methods. The MBD methods, on the other hand, have not yet been extended beyond the dipole approximation. The inclusion of higher multipoles would be beneficial for two reasons. First, it should improve the accuracy of the vdW model for strongly polarizable systems at equilibrium geometries. Second, it would enable to systematically study a whole new class of vdW effects such as the dependence of vdW interactions on external electric fields, which cannot be done within the dipole approximation.

Acknowledgments The authors acknowledge partial financial support by the Luxembourg National Research Fund within the FNR-CORE program (No. FNR-11360857) and the ERC Consolidator Grant "BeStMo." 


\section{References}

Ambrosetti A, Alfè D, DiStasio RA Jr, Tkatchenko A (2014a) Hard numbers for large molecules: toward exact energetics for supramolecular systems. J Phys Chem Lett 5(5):849-855. https:// doi.org/10.1021/jz402663k

Ambrosetti A, Reilly AM, DiStasio RA Jr, Tkatchenko A (2014b) Long-range correlation energy calculated from coupled atomic response functions. J Chem Phys 140(18):18A508. https://doi. org/10.1063/1.4865104

Andersson Y, Langreth DC, Lundqvist BI (1996) Van der Waals interactions in density-functional theory. Phys Rev Lett 76(1):102-105. https://doi.org/10.1103/PhysRevLett.76.102

Ángyán JG (2007) On the exchange-hole model of London dispersion forces. J Chem Phys 127(2):024,108. https://doi.org/10.1063/1.2749512

Ángyán JG, Liu RF, Toulouse J, Jansen G (2011) Correlation energy expressions from the adiabatic-connection fluctuation-dissipation theorem approach. J Chem Theory Comput 7(10):3116-3130. https://doi.org/10.1021/ct200501r

Axilrod BM, Teller E (1943) Interaction of the van der Waals type between three atoms. J Chem Phys 11(6):299-300. https://doi.org/10.1063/1.1723844

Ayers PW (2009) A perspective on the link between the exchange(-correlation) hole and dispersion forces. J Math Chem 46(1):86-96. https://doi.org/10.1007/s10910-008-9451-y

Bade WL (1957) Drude-model calculation of dispersion forces. I. General theory. J Chem Phys 27(6):1280-1284. https://doi.org/10.1063/1.1743991

Bade WL, Kirkwood JG (1957) Drude-model calculation of dispersion forces. II. The linear lattice. J Chem Phys 27(6):1284-1288. https://doi.org/10.1063/1.1743992

Becke AD (1988) A multicenter numerical integration scheme for polyatomic molecules. J Chem Phys 88(4):2547-2553. https://doi.org/10.1063/1.454033

Becke AD, Johnson ER (2005a) A density-functional model of the dispersion interaction. J Chem Phys 123(15):154,101. https://doi.org/10.1063/1.2065267

Becke AD, Johnson ER (2005b) Exchange-hole dipole moment and the dispersion interaction. J Chem Phys 122(15):154,104. https://doi.org/10.1063/1.1884601

Becke AD, Johnson ER (2006) Exchange-hole dipole moment and the dispersion interaction: highorder dispersion coefficients. J Chem Phys 124(1):014,104. https://doi.org/10.1063/1.2139668

Becke AD, Roussel MR (1989) Exchange holes in inhomogeneous systems: a coordinate-space model. Phys Rev A 39(8):3761-3767. https://doi.org/10.1103/PhysRevA.39.3761

Björkman T (2012) Van der Waals density functional for solids. Phys Rev B 86(16):165,109. https://doi.org/10.1103/PhysRevB.86.165109

Björkman T (2014) Testing several recent van der Waals density functionals for layered structures. J Chem Phys 141(7):074,708. https://doi.org/10.1063/1.4893329

Björkman T, Gulans A, Krasheninnikov AV, Nieminen RM (2012) Are we van der Waals ready? J Phys Condens Matter 24(42):424,218. https://doi.org/10.1088/0953-8984/24/42/424218

Brandenburg JG, Grimme S (2014) Accurate modeling of organic molecular crystals by dispersioncorrected density functional tight binding (DFTB). J Phys Chem Lett 5(11):1785-1789. https:// doi.org/10.1021/jz500755u

Bučko T, Lebègue S, Hafner J, Ángyán JG (2013) Improved density dependent correction for the description of London dispersion forces. J Chem Theory Comput 9(10):4293-4299. https://doi. org/10.1021/ct400694h

Bučko T, Lebègue S, Ángyán JG, Hafner J (2014) Extending the applicability of the TkatchenkoScheffler dispersion correction via iterative Hirshfeld partitioning. J Chem Phys 141(3):034,114. https://doi.org/10.1063/1.4890003

Bultinck P, Alsenoy CV, Ayers PW, Carbó-Dorca R (2007) Critical analysis and extension of the Hirshfeld atoms in molecules. J Chem Phys 126(14):144,111. https://doi.org/10.1063/1. 2715563

Callen HB, Welton TA (1951) Irreversibility and generalized noise. Phys Rev 83(1):34-40. https:// doi.org/10.1103/PhysRev.83.34 
Dion M, Rydberg H, Schröder E, Langreth DC, Lundqvist BI (2004) Van der Waals density functional for general geometries. Phys Rev Lett 92(24):246,401. https://doi.org/10.1103/ PhysRevLett.92.246401

Dobson JF, White A, Rubio A (2006) Asymptotics of the dispersion interaction: analytic benchmarks for van der Waals energy functionals. Phys Rev Lett 96(7):073,201. https://doi. org/10.1103/PhysRevLett.96.073201

Donchev AG (2006) Many-body effects of dispersion interaction. J Chem Phys 125(7):074,713. https://doi.org/10.1063/1.2337283

Elstner M, Hobza P, Frauenheim T, Suhai S, Kaxiras E (2001) Hydrogen bonding and stacking interactions of nucleic acid base pairs: a density-functional-theory based treatment. J Chem Phys 114(12):5149-5155. https://doi.org/10.1063/1.1329889

Freysoldt C, Grabowski B, Hickel T, Neugebauer J, Kresse G, Janotti A, Van de Walle CG (2014) First-principles calculations for point defects in solids. Rev Mod Phys 86(1):253-305. https:// doi.org/10.1103/RevModPhys.86.253

Gao W, Tkatchenko A (2013) Electronic structure and van der Waals interactions in the stability and mobility of point defects in semiconductors. Phys Rev Lett 111(4):045,501. https://doi.org/ 10.1103/PhysRevLett.111.045501

Ge X, Lu D (2015) Local representation of the electronic dielectric response function. Phys Rev B 92(24):241,107. https://doi.org/10.1103/PhysRevB.92.241107

Geim AK, Grigorieva IV (2013) Van der Waals heterostructures. Nature 499(7459):419-425. https://doi.org/10.1038/nature12385

Gell-Mann M, Brueckner KA (1957) Correlation energy of an electron gas at high density. Phys Rev 106(2):364-368. https://doi.org/10.1103/PhysRev.106.364

Gobre VV, Tkatchenko A (2013) Scaling laws for van der Waals interactions in nanostructured materials. Nat Commun 4:2341. https://doi.org/10.1038/ncomms3341

Grimme S (2004) Accurate description of van der Waals complexes by density functional theory including empirical corrections. J Comput Chem 25(12):1463-1473. https://doi.org/10.1002/ jcc. 20078

Grimme S (2006) Semiempirical GGA-type density functional constructed with a long-range dispersion correction. J Comput Chem 27(15):1787-1799. https://doi.org/10.1002/jcc.20495

Grimme S, Antony J, Ehrlich S, Krieg H (2010) A consistent and accurate ab initio parametrization of density functional dispersion correction (DFT-D) for the 94 elements H-Pu. J Chem Phys 132(15):154,104. https://doi.org/10.1063/1.3382344

Gunnarsson O, Lundqvist BI (1976) Exchange and correlation in atoms, molecules, and solids by the spin-density-functional formalism. Phys Rev B 13(10):4274-4298. https://doi.org/10.1103/ PhysRevB.13.4274

Halgren TA (1992) The representation of van der Waals (vdW) interactions in molecular mechanics force fields: potential form, combination rules, and vdW parameters. J Am Chem Soc 114(20):7827-7843. https://doi.org/10.1021/ja00046a032

Harl J, Schimka L, Kresse G (2010) Assessing the quality of the random phase approximation for lattice constants and atomization energies of solids. Phys Rev B 81(11):115,126. https://doi.org/ 10.1103/PhysRevB.81.115126

Hepburn J, Scoles G, Penco R (1975) A simple but reliable method for the prediction of intermolecular potentials. Chem Phys Lett 36(4):451-456. https://doi.org/10.1016/00092614(75) 80278-8

Hermann J, DiStasio RA Jr, Tkatchenko A (2017) First-principles models for van der Waals interactions in molecules and materials: concepts, theory, and applications. Chem Rev 117(6): 4714-4758. https://doi.org/10.1021/acs.chemrev.6b00446

Heßelmann A (2009) Derivation of the dispersion energy as an explicit density- and exchange-hole functional. J Chem Phys 130(8):084,104. https://doi.org/10.1063/1.3077939

Hirshfeld FL (1977) Bonded-atom fragments for describing molecular charge densities. Theoret Chim Acta 44(2):129-138. https://doi.org/10.1007/BF00549096

Hunt KLC (1983) Nonlocal polarizability densities and van der Waals interactions. J Chem Phys 78(10):6149-6155. https://doi.org/10.1063/1.444577 
Jansen G, Liu RF, Ángyán JG (2010) On the equivalence of ring-coupled cluster and adiabatic connection fluctuation-dissipation theorem random phase approximation correlation energy expressions. J Chem Phys 133(15):154,106. https://doi.org/10.1063/1.3481575

Johnson ER, Becke AD (2006) A post-Hartree-Fock model of intermolecular interactions: inclusion of higher-order corrections. J Chem Phys 124(17):174,104. https://doi.org/10.1063/ 1.2190220

Jurečka P, Šponer J, Černý J, Hobza P (2006) Benchmark database of accurate (MP2 and CCSD(T) complete basis set limit) interaction energies of small model complexes, DNA base pairs, and amino acid pairs. Phys Chem Chem Phys 8(17):1985-1993. https://doi.org/10.1039/B600027D

Kohn W, Meir Y, Makarov DE (1998) Van der Waals energies in density functional theory. Phys Rev Lett 80(19):4153-4156. https://doi.org/10.1103/PhysRevLett.80.4153

Kurth S, Perdew JP (1999) Density-functional correction of random-phase-approximation correlation with results for jellium surface energies. Phys Rev B 59(16):10,461-10,468. https://doi. org/10.1103/PhysRevB.59.10461

Landau LD, Lifschitz EM (1980) Statistical physics, vol 5, 3rd edn. Pergamon Press, Oxford

Langbein D (1974) Theory of Van Der Waals attraction, Springer tracts in modern physics, vol 72. Springer, Berlin/Heidelberg. https://doi.org/10.1007/BFb0042407. URL http://link.springer. com/chapter/10.1007/BFb0042407

Langreth DC, Perdew JP (1977) Exchange-correlation energy of a metallic surface: wave-vector analysis. Phys Rev B 15(6):2884-2901. https://doi.org/10.1103/PhysRevB.15.2884

Lein M, Gross EKU, Perdew JP (2000) Electron correlation energies from scaled exchangecorrelation kernels: importance of spatial versus temporal nonlocality. Phys Rev B 61(20):13,431-13,437. https://doi.org/10.1103/PhysRevB.61.13431

Lucas A (1967) Collective contributions to the long-range dipolar interaction in rare-gas crystals. Physica 35(3):353-368. https://doi.org/10.1016/0031-8914(67)90184-X

Mahan GD (1965) Van der Waals forces in solids. J Chem Phys 43(5):1569-1574. https://doi.org/ $10.1063 / 1.1696973$

Margenau H (1939) Van der Waals forces. Rev Mod Phys 11(1):1-35. https://doi.org/10.1103/ RevModPhys.11.1

Marzari N, Mostofi AA, Yates JR, Souza I, Vanderbilt D (2012) Maximally localized Wannier functions: theory and applications. Rev Mod Phys 84(4):1419-1475. https://doi.org/10.1103/ RevModPhys.84.1419

Mayer A (2007) Formulation in terms of normalized propagators of a charge-dipole model enabling the calculation of the polarization properties of fullerenes and carbon nanotubes. Phys Rev B 75(4):045,407. https://doi.org/10.1103/PhysRevB.75.045407

Mooij WTM, van Duijneveldt FB, van Duijneveldt-van de Rijdt JGCM, van Eijck BP (1999) Transferable ab initio intermolecular potentials. 1. Derivation from methanol dimer and trimer calculations. J Phys Chem A 103(48):9872-9882. https://doi.org/10.1021/jp991641n

Mutō Y (1943) Hikyokusei bunshi no aida ni sayō suru chikara ni tsuite [On forces acting between nonpolar molecules]. Nippon Sugaku Buturigakkwaishi 17:629-631. https://doi.org/10.11429/ subutsukaishi1927.17.10-11-12_629

Olsen T, Thygesen KS (2012) Extending the random-phase approximation for electronic correlation energies: the renormalized adiabatic local density approximation. Phys Rev B 86(8):081,103. https://doi.org/10.1103/PhysRevB.86.081103

Olsen T, Thygesen KS (2013a) Beyond the random phase approximation: improved description of short-range correlation by a renormalized adiabatic local density approximation. Phys Rev B 88(11):115,131. https://doi.org/10.1103/PhysRevB.88.115131

Olsen T, Thygesen KS (2013b) Random phase approximation applied to solids, molecules, and graphene-metal interfaces: from van der Waals to covalent bonding. Phys Rev B 87(7):075,111. https://doi.org/10.1103/PhysRevB.87.075111

Olsen T, Thygesen KS (2014) Accurate ground-state energies of solids and molecules from timedependent density-functional theory. Phys Rev Lett 112(20):203,001. https://doi.org/10.1103/ PhysRevLett.112.203001 
Otero-de-la-Roza A, Johnson ER (2012) A benchmark for non-covalent interactions in solids. J Chem Phys 137(5):054,103. https://doi.org/10.1063/1.4738961

Parr RG, Yang W (1989) Density-functional theory of atoms and molecules. No. 16 in The international series of monographs on chemistry. Oxford University Press. URL https://global.oup. com/academic/product/density-functional-theory-of-atoms-and-molecules-9780195092769

Parsegian VA (2005) Van der Waals forces: a handbook for biologists, chemists, engineers, and physicists. Cambridge University Press, Cambridge. URL http://ebooks.cambridge.org/ref/id/ CBO9780511614606

Reilly AM, Tkatchenko A (2013a) Seamless and accurate modeling of organic molecular materials. J Phys Chem Lett 4(6):1028-1033. https://doi.org/10.1021/jz400226x

Reilly AM, Tkatchenko A (2013b) Understanding the role of vibrations, exact exchange, and many-body van der Waals interactions in the cohesive properties of molecular crystals. J Chem Phys 139(2):024,705. https://doi.org/10.1063/1.4812819

Reilly AM, Cooper RI, Adjiman CS, Bhattacharya S, Boese AD, Brandenburg JG, Bygrave PJ, Bylsma R, Campbell JE, Car R, Case DH, Chadha R, Cole JC, Cosburn K, Cuppen HM, Curtis F, Day GM, DiStasio RA Jr, Dzyabchenko A, van Eijck BP, Elking DM, van den Ende JA, Facelli JC, Ferraro MB, Fusti-Molnar L, Gatsiou CA, Gee TS, de Gelder R, Ghiringhelli LM, Goto H, Grimme S, Guo R, Hofmann DWM, Hoja J, Hylton RK, Iuzzolino L, Jankiewicz W, de Jong DT, Kendrick J, de Klerk NJJ, Ko HY, Kuleshova LN, Li X, Lohani S, Leusen FJJ, Lund AM, Lv J, Ma Y, Marom N, Masunov AE, McCabe P, McMahon DP, Meekes H, Metz MP, Misquitta AJ, Mohamed S, Monserrat B, Needs RJ, Neumann MA, Nyman J, Obata S, Oberhofer H, Oganov AR, Orendt AM, Pagola GI, Pantelides CC, Pickard CJ, Podeszwa R, Price LS, Price SL, Pulido A, Read MG, Reuter K, Schneider E, Schober C, Shields GP, Singh P, Sugden IJ, Szalewicz K, Taylor CR, Tkatchenko A, Tuckerman ME, Vacarro F, Vasileiadis M, Vazquez-Mayagoitia A, Vogt L, Wang Y, Watson RE, de Wijs GA, Yang J, Zhu Q, Groom CR (2016) Report on the sixth blind test of organic crystal structure prediction methods. Acta Cryst B 72(4):439-459. https://doi.org/10.1107/S2052520616007447

Ren X, Tkatchenko A, Rinke P, Scheffler M (2011) Beyond the random-phase approximation for the electron correlation energy: the importance of single excitations. Phys Rev Lett 106(15):153,003. https://doi.org/10.1103/PhysRevLett.106.153003

Ren X, Rinke P, Joas C, Scheffler M (2012) Random-phase approximation and its applications in computational chemistry and materials science. J Mater Sci 47(21):7447-7471. https://doi.org/ 10.1007/s10853-012-6570-4

Ren X, Rinke P, Scuseria GE, Scheffler M (2013) Renormalized second-order perturbation theory for the electron correlation energy: concept, implementation, and benchmarks. Phys Rev B 88(3):035,120. https://doi.org/10.1103/PhysRevB.88.035120

Renne MJ, Nijboer BRA (1967) Microscopic derivation of macroscopic Van der Waals forces. Chem Phys Lett 1(8):317-320. https://doi.org/10.1016/0009-2614(67)80004-6

Řezáč J, Riley KE, Hobza P (2011) S66: a well-balanced database of benchmark interaction energies relevant to biomolecular structures. J Chem Theory Comput 7(8):2427-2438. https:// doi.org/10.1021/ct2002946

Ruiz VG, Liu W, Zojer E, Scheffler M, Tkatchenko A (2012) Density-functional theory with screened van der Waals interactions for the modeling of hybrid inorganic-organic systems. Phys Rev Lett 108(14):146,103. https://doi.org/10.1103/PhysRevLett.108.146103

Ruzsinszky A, Perdew JP, Tao J, Csonka GI, Pitarke JM (2012) Van der Waals coefficients for nanostructures: fullerenes defy conventional wisdom. Phys Rev Lett 109(23):233,203. https:// doi.org/10.1103/PhysRevLett.109.233203

Sato T, Nakai H (2009) Density functional method including weak interactions: dispersion coefficients based on the local response approximation. J Chem Phys 131(22):224,104. https:// doi.org/10.1063/1.3269802

Sato T, Nakai H (2010) Local response dispersion method. II. Generalized multicenter interactions. J Chem Phys 133(19):194,101. https://doi.org/10.1063/1.3503040

Schimka L, Gaudoin R, Klimeš J, Marsman M, Kresse G (2013) Lattice constants and cohesive energies of alkali, alkaline-earth, and transition metals: random phase approximation and den- 
sity functional theory results. Phys Rev B 87(21):214,102. https://doi.org/10.1103/PhysRevB. 87.214102

Scuseria GE, Henderson TM, Sorensen DC (2008) The ground state correlation energy of the random phase approximation from a ring coupled cluster doubles approach. J Chem Phys 129(23):231,101. https://doi.org/10.1063/1.3043729

Silvestrelli PL (2008) Van der Waals interactions in DFT made easy by Wannier functions. Phys Rev Lett 100(5):053,002. https://doi.org/10.1103/PhysRevLett.100.053002

Silvestrelli PL (2013) Van der Waals interactions in density functional theory by combining the quantum harmonic oscillator-model with localized Wannier functions. J Chem Phys 139(5):054,106. https://doi.org/10.1063/1.4816964

Silvestrelli PL, Benyahia K, Grubisiĉ S, Ancilotto F, Toigo F (2009) Van der Waals interactions at surfaces by density functional theory using Wannier functions. J Chem Phys 130(7):074,702. https://doi.org/10.1063/1.3077288

Steinmann SN, Corminboeuf C (2010) A system-dependent density-based dispersion correction. J Chem Theory Comput 6(7):1990-2001. https://doi.org/10.1021/ct1001494

Steinmann SN, Corminboeuf C (2011) Comprehensive benchmarking of a density-dependent dispersion correction. J Chem Theory Comput 7(11):3567-3577. https://doi.org/10.1021/ ct $200602 x$

Stone AJ (2013) The theory of intermolecular forces, 2nd edn. Oxford University Press, Oxford URL http://www.oxfordscholarship.com/view/10.1093/acprof:oso/9780199672394.001.0001/ acprof-9780199672394

Szabo A, Ostlund NS (1996) Modern quantum chemistry: introduction to advanced electronic structure theory, rev edn. URL http://store.doverpublications.com/0486691861.html

Tao J, Perdew JP, Ruzsinszky A (2010) Long-range van der Waals attraction and alkali-metal lattice constants. Phys Rev B 81(23):233,102. https://doi.org/10.1103/PhysRevB.81.233102

Tkatchenko A, Scheffler M (2009) Accurate molecular Van Der Waals interactions from groundstate electron density and free-atom reference data. Phys Rev Lett 102(7):073,005. https://doi. org/10.1103/PhysRevLett.102.073005

Tkatchenko A, DiStasio RA Jr, Car R, Scheffler M (2012) Accurate and efficient method for many-body van der Waals interactions. Phys Rev Lett 108(23):236,402. https://doi.org/10.1103/ PhysRevLett.108.236402

Tkatchenko A, Ambrosetti A, DiStasio RA Jr (2013) Interatomic methods for the dispersion energy derived from the adiabatic connection fluctuation-dissipation theorem. J Chem Phys 138(7):74,106. https://doi.org/10.1063/1.4789814

Toulouse J, Colonna F, Savin A (2004) Long-range-short-range separation of the electron-electron interaction in density-functional theory. Phys Rev A 70(6):062,505. https://doi.org/10.1103/ PhysRevA.70.062505

Toulouse J, Gerber IC, Jansen G, Savin A, Ángyán JG (2009) Adiabatic-connection fluctuationdissipation density-functional theory based on range separation. Phys Rev Lett 102(9):096,404. https://doi.org/10.1103/PhysRevLett.102.096404

Unsöld A (1927) Quantentheorie des Wasserstoffmolekülions und der Born-Landéschen Abstoßungskräfte. Z Physik 43(8):563-574. https://doi.org/10.1007/BF01397633

Verstraelen T, Pauwels E, De Proft F, Van Speybroeck V, Geerlings P, Waroquier M (2012) Assessment of atomic charge models for gas-phase computations on polypeptides. J Chem Theory Comput 8(2):661-676. https://doi.org/10.1021/ct200512e

Vydrov OA, Van Voorhis T (2010) Nonlocal van der Waals density functional: the simpler the better. J Chem Phys 133(24):244,103. https://doi.org/10.1063/1.3521275

Wu Q, Yang W (2002) Empirical correction to density functional theory for van der Waals interactions. J Chem Phys 116(2):515-524. https://doi.org/10.1063/1.1424928

Zhang GX, Tkatchenko A, Paier J, Appel H, Scheffler M (2011) Van der Waals interactions in ionic and semiconductor solids. Phys Rev Lett 107(24):245,501. https://doi.org/10.1103/ PhysRevLett.107.245501 